\title{
Fatigue Damage Evaluation of Broad-Band Gaussian and Non-Gaussian Wind Load Effects by a Spectral Method
}

\author{
Jie Ding, Xinzhong Chen \\ National Wind Institute, Department of Civil and Environmental Engineering, Texas Tech University, TX \\ Emails: jie.ding@ttu.edu; xinzhong.chen@ttu.edu
}

\begin{abstract}
:
This study presents fatigue damage evaluation of broad-band Gaussian and non-Gaussian wind load effects by a spectral method proposed in Benasciutti and Tovo (2005). The wind load effects considered are alongwind, crosswind and their coupled responses of tall buildings, and wind pressures on claddings. Following this spectral method, the rainflow counting damage is approximated by a linear combination of its upper and lower bounds. A refined formulation for determining the combination factor is proposed, which depends on bandwidth parameters in terms of process spectral moments. For non-Gaussian wind load effects, research emphasis is placed on the modeling of translation function which relates the nonGaussian process with an underlying Gaussian process and is essential for non-Gaussian fatigue damage evaluation. Both moment-based translation model and the model by a direct curve fitting are addressed for a wide range of non-Gaussian characteristics, including a newly developed translation model for hardening non-Gaussian processes. The effectiveness and accuracy of the spectral method for broad-band Gaussian and non-Gaussian processes are demonstrated through comparison with the results from time domain rainflow counting method.
\end{abstract}

Keywords: Fatigue damage evaluation; Rainflow cycle counting; Non-Gaussian fatigue; Spectral method; Tall buildings; Roof claddings; Wind load effects. 


\section{Introduction}

Stochastic wind load effects on structures may lead to accumulation of fatigue damage and result in failure of structural components and system. The wind-induced fatigue and extreme load effects are two limit-state responses important for design consideration of wind-excited structures (Lynn and Stathopoulos 1985; Xu 1995; Simiu and Scanlan 1996; Holmes 2002; Repetto and Solari 2004 and 2006; Ding and Chen 2013; Chen 2014a and b).

When the stress time history is available, time domain approaches can be applied for fatigue damage evaluation. The cycle number as a function of stress amplitude, often referred to as fatigue loading spectrum, is estimated using a cycle counting method. The cumulative fatigue damage is then determined using Palmgren-Miner rule with S-N curve. A number of approaches have been developed for cycle counting, including peak counting, level-crossing counting, range-mean counting and rainflow counting methods (ASTM 1049-85; Dowling 1972; Rychlik 1993). Among them, the rainflow counting method provides a better estimation for general stochastic processes, and has been accepted as a standard cycle counting method in fatigue analysis (e.g., ASTM 1049-85; Xu 1995; Kumar and Stathopoulos 1998). However, the construction of a reliable cycle distribution relies on a sufficient length of stress time history, which is not always available in engineering practice.

Alternatively, the cycle counting and fatigue damage analysis can be performed using frequency domain approaches, which develop analytical formulae from process power spectral density (PSD) function. In the case of narrow-band Gaussian processes, the cycle distribution is readily determined from Rayleigh distribution of process amplitude, and the fatigue damage is then calculated by a closed-form expression. However, the fatigue damage from this approximation can be very conservative as compared to the rainflow counting damage when the stress process is not narrow-banded. Various approaches have been developed for broad-band Gaussian processes to approximate rainflow counting damage taking into account the spectral properties (Wirsching and Light 1980; Lutes and Larsen 1990; Zhao and Baker 1990; Tovo 2002; Gao and Moan 2008). For instance, a correction factor was introduced by Wirsching and 
Light (1980) to characterize the difference between narrow-band approximation and rainflow counting method. Another simple correction was proposed by Lutes and Larsen (1990), which is referred to as single-moment method since the correction depends on only one spectral moment. The method proposed by Jiao and Moan (1990) was for broad-band Gaussian processes with low-frequency and high-frequency bimodal spectral formulation. This method has been refined by Gao and Moan (2008) for Gaussian processes with a trimodal spectrum. Directly seeking an approximation to rainflow amplitudes was attempted by several researchers (e.g., Dirlik 1985; Zhao and Baker 1990; Bouyssy et al. 1993), among which the most accurate and wildly used empirical formulations are considered to be the ones proposed by Dirlik (1985), Zhao and Baker (1990). Tovo (2002) provided another approximation to rainflow counting damage, which estimates the fatigue damage as a proper intermediate point between its upper and lower bounds defined by level-crossing counting and range-mean counting methods, respectively. The advantage of this method is that an equivalent joint probability distribution function (JPDF) of peak and valley can be obtained (Benasciutti and Tovo 2006). It facilitates further considerations of mean stress effect and also non-Gaussian characteristics of a stress process in fatigue damage evaluation.

Spectral methods for fatigue analysis of non-Gaussian processes have also been addressed in literature (Lutes et al. 1984; Winterstein 1988; Benasciutti and Tovo 2005; Gao and Moan 2007), primarily focusing on softening non-Gaussian processes with kurtosis larger than 3 , which lead to accelerated fatigue damage than Gaussian processes. The non-Gaussian process can be modeled as a translation process from an underlying Gaussian process through a monotonic increasing translation function (Grigoriu 1984 and 1995; Winterstein 1988). Subsequently, the occurrence of peak and valley in the underlying Gaussian process is always simultaneous as in the non-Gaussian process due to the monotone property of translation. Therefore, the distribution of peak and valley for the non-Gaussian process can be determined if the one for its underlying Gaussian process is specified. In the case of narrow-band unskewed non-Gaussian processes, a closed-from correction as a function of process kurtosis was introduced for the fatigue analysis from that of underlying Gaussian process (Winterstein 1988; Lutes and Sarkani 2004). A more accurate correction was recently introduced in Chen (2014b) for 
narrow-band unskewed hardening non-Gaussian processes with kurtosis less than 3. The accuracy and effectiveness of the fatigue analysis methods for non-Gaussian processes based on translation process theory depend on the adequacy of the equivalent JPDF of peak and valley of underlying Gaussian process and the modeling of translation function. Considering various broad-band spectral properties and nonGaussian distribution characteristics, the spectral methods for fatigue analysis of broad-band nonGaussian processes warrant further investigation.

Concerning the fatigue damage analysis of wind-excited structures, Homles (2002) derived closedform expressions for upper and lower limits of fatigue damage of alongwind response. The upper limit was achieved by narrow-band approximation, while the lower one was obtained based on the correction factor proposed by Wirshing and Light (1980). However, as pointed out by Lutes et al. (1984) and Repetto and Solari (2006), the damage correction factor proposed by Wirshing and Light (1980) may underestimate or overestimate the real damage, depending on the spectral shape of the stress process. Repetto and Solari (2004) addressed fatigue damage of coupled alongwind and crosswind response using the approach developed by Jiao and Moan (1990). The fatigue damage of alongwind response with both broad-band background (quasi-static) response and narrow-band resonant response components was studied in Repetto and Solari (2006). While the fatigue damage of broad-band wind load effects on roof claddings have been investigated using rainflow counting method (Xu 1995; Kumar and Stathopoulos 1998), the adequacy of the spectral methods for fatigue damage associated with broad-band Gaussian and non-Gaussian wind load effects has not yet been extensively explored. Full-scale and wind tunnel measurement data have shown that the wind pressures on claddings may be of significant non-Gaussian characteristics (e.g., Holmes 1981; Yeatts and Mehta 1993; Kumar and Stathopoulos 1998). The application of translation process theory to non-Gaussian wind pressures faces many challenges in better modeling the translation functions and their extreme value distributions (Ding and Chen 2014). Similar challenges are expected for the fatigue analysis of broad-band non-Gaussian processes in addition to the influence of spectral properties. 
In this study, the spectral method introduced in Benasciutti and Tovo (2005), referred to as TB spectral method, is re-evaluated for fatigue analysis of broad-band Gaussian and non-Gaussian wind load effects. The wind load effects considered are alongwind, crosswind and their coupled responses of tall buildings, and wind pressures on claddings. The accuracy of the TB spectral method is examined through comparison with rainflow counting damage estimated from sufficient long time history samples. A refined formulation is proposed to account for the influence of spectral shape on fatigue damage. For nonGaussian wind load effects, research emphasis is placed on the modeling of translation function which relates the non-Gaussian process to an underlying Gaussian process and is essential for non-Gaussian fatigue damage evaluation. The results demonstrate the effectiveness and accuracy of the spectral method developed for broad-band Gaussian and non-Gaussian processes.

\section{Spectral method for fatigue analysis of Gaussian processes}

\subsection{Narrow-band Gaussian processes}

For a very narrow-band stress process $X(t)$, it is reasonable to state that a stress cycle is formed by a peak and the following symmetrical valley, and the amplitude equals to the value of peak if the process is of zero mean. Therefore, the peak counting, level-crossing counting, range-mean counting and rainflow counting methods result in the same cycle distribution. Furthermore, the probability distribution of amplitude (or range) is identical to that of the peaks, and follows a Rayleigh distribution:

$$
p(s)=\frac{s}{\sigma_{X}^{2}} e^{-\frac{s^{2}}{2 \sigma_{X}^{2}}}
$$

where $s$ is the stress amplitude; and $\sigma_{X}$ is standard deviation (STD) of the stress process.

The number of cycles per unit time $\left(v_{a}\right)$ can be taken as the occurrence rate of upcrossings at mean level $\left(v_{0}\right)$, then the mean fatigue damage per unit time, i.e., mean damage rate $\left(E\left[D_{N B}\right]\right)$, is estimated as (Madsen et al. 1986)

$$
E\left[D_{N B}\right]=v_{a} C^{-1} \int_{0}^{\infty} s^{k} p(s) d s=v_{0} C^{-1}\left(\sqrt{2} \sigma_{X}\right)^{k} \Gamma\left(1+\frac{k}{2}\right)
$$


where the S-N curve defined as $N(s)=C s^{-k}$ is applied; $\Gamma()$ is the gamma function defined as $\Gamma(t)=$ $\int_{0}^{\infty} x^{t-1} e^{-x} \mathrm{~d} x ; v_{0}$ can be determined from spectral moments as

$$
\begin{gathered}
v_{0}=\frac{1}{2 \pi} \sqrt{\frac{\lambda_{2}}{\lambda_{0}}} \\
\lambda_{n}=\int_{0}^{\infty}(2 \pi f)^{n} S_{X}(f) d f
\end{gathered}
$$

and $\lambda_{n}$ is $n$-th moment of the process $\operatorname{PSD}, S_{X}(f)$; and $f$ is frequency in $\mathrm{Hz}$.

\subsection{Broad-band Gaussian processes}

It is well-known that for a broad-band Gaussian process, the fatigue damage estimated from Rayleigh distribution of amplitude (narrow-band approximation) is very conservative with comparison to rainflow counting damage (Rychlik 1993). However, an explicit analytical distribution function for rainflow cycles of a broad-band process is not available so far due to the complex definition of this algorithm (Dowling 1972). In this section, the approach introduced by Tovo (2002), and Benasciutti and Tovo (2005), referred to as TB spectral method, is discussed, which provides an equivalent JPDF of peak and valley for rainflow counting method, and facilitates its application to non-Gaussian processes.

For a stationary Gaussian process, it is widely accepted that the fatigue damage rate calculated from rainflow counting method, $E\left[D_{R F}\right]$, is always bounded by that from level-crossing counting and rangemean counting methods, $E\left[D_{L C}\right]$ and $E\left[D_{R M}\right]$, as (Madsen et al. 1986; Rychlik 1993; Tovo 2002)

$$
\begin{gathered}
E\left[D_{R M}\right] \leq E\left[D_{R F}\right] \leq E\left[D_{L C}\right] \\
E\left[D_{L C}\right]=v_{p} C^{-1} \alpha_{2}\left(\sqrt{2} \sigma_{X}\right)^{k} \Gamma\left(1+\frac{k}{2}\right)=E\left[D_{N B}\right] \\
E\left[D_{R M}\right]=v_{p} C^{-1}\left(\sqrt{2} \sigma_{X} \alpha_{2}\right)^{k} \Gamma\left(1+\frac{k}{2}\right)=\alpha_{2}^{k-1} E\left[D_{L C}\right]
\end{gathered}
$$

where $v_{p}$ is the occurrence rate of peak and $\alpha_{2}$ is a bandwidth parameter, which are estimated from spectral moments as 


$$
\begin{gathered}
v_{p}=\frac{1}{2 \pi} \sqrt{\frac{\lambda_{4}}{\lambda_{2}}} \\
\alpha_{n}=\frac{\lambda_{n}}{\sqrt{\lambda_{0} \lambda_{2 n}}}
\end{gathered}
$$

where $0 \leq \alpha_{n} \leq 1$, and $\alpha_{n}$ tends to be unity for a narrow-band process. Comparing Eqs. (8) and (9) with Eq. (3), we can get $v_{0}=\alpha_{2} v_{p}$.

Based on a comprehensive analysis for a variety of broad-band processes with different spectral shapes, a linear combination rule to approximate the rainflow counting damage rate of a broad-band process was proposed in Tovo (2002) and Benasciutti and Tovo (2005) as

$$
\begin{gathered}
E\left[D_{R F}\right]=b E\left[D_{L C}\right]+(1-b) E\left[D_{R M}\right] \\
b=\frac{\left(\alpha_{1}-\alpha_{2}\right)\left[1.112\left(1+\alpha_{1} \alpha_{2}-\left(\alpha_{1}+\alpha_{2}\right)\right) e^{2.11 \alpha_{2}}+\left(\alpha_{1}-\alpha_{2}\right)\right]}{\left(\alpha_{2}-1\right)^{2}}
\end{gathered}
$$

It is noted that the substitution of Eqs. (6) and (7) into Eq. (10) yields

$$
E\left[D_{R F}\right]=\left[b+(1-b) \alpha_{2}^{k-1}\right] E\left[D_{N B}\right]
$$

Apparently, the TB spectral method also provides a correction factor for the approximation of rainflow counting damage from narrow-band approximation. The correction factor is a function of two nondimensional parameters $\alpha_{1}$ and $\alpha_{2}$, which are calculated by four spectral moments $\lambda_{0}, \lambda_{1}, \lambda_{2}$ and $\lambda_{4}$.

If the equivalent JPDF of peak $(u)$ and valley $(v)$ is denoted as $h(u, v)$, the JPDF of amplitude $s=\frac{1}{2}(u-v)$ and mean $m=\frac{1}{2}(u+v), f(s, m)$, can be expressed as

$$
f(s, m)=2 h(m+s, m-s)
$$

The amplitude distribution is the marginal of this joint distribution

$$
p(s)=\int_{-\infty}^{+\infty} f(s, m) d m
$$

and the damage rate is subsequently estimated as

$$
E[D]=v_{a} C^{-1} \int_{0}^{\infty} s^{k} p(s) d s=v_{a} C^{-1} \int_{-\infty}^{\infty} \int_{-\infty}^{\infty}\left(\frac{u-v}{2}\right)^{k} h(u, v) d v d u
$$


For rainflow counting method, every peak is associated with a counted cycle (Rychlik 1993), thus $v_{a}=v_{p}$. As the relation between cycle distribution and fatigue damage is a linear summation (i.e., Palmgren-Miner rule), a similar combination rule as defined by Eq. (10) is expected for cycle distribution (Benasciutti and Tovo 2005). An equivalent JPDF of peak and the following valley associated with rainflow counting damage, i.e., $h_{R F}(u, v)$, can be postulated in the form (Benasciutti and Tovo 2005)

$$
h_{R F}(u, v)=b h_{L C}(u, v)+(1-b) h_{R M}(u, v)
$$

where $h_{L C}(u, v)$ and $h_{R M}(u, v)$ are JPDFs of peak and valley associated with level-crossing counting and rang-mean counting methods. It should be noted that $h_{R F}(u, v), h_{L C}(u, v)$ and $h_{R M}(u, v)$ are only for $u \geq v$

The equivalent JPDF of peak and valley for $D_{L C}$ can be expressed as follows (Tovo 2002)

$$
h_{L C}(u, v)= \begin{cases}{\left[p_{u}(u)-p_{v}(u)\right] \delta(u+v)+p_{v}(u) \delta(u-v)} & u>0 \\ p_{u}(u) \delta(u-v) & u \leq 0\end{cases}
$$

where $\delta$ is Dirac delta function; $p_{u}(x)$ and $p_{v}(x)$ are PDFs of peak and valley of a process $X(t)$. For a zero-mean Gaussian process, $p_{u}(x)$ is given as

$$
p_{u}(x)=\frac{\sqrt{1-\alpha_{2}^{2}}}{\sqrt{2 \pi} \sigma_{X}} e^{-\frac{x^{2}}{2 \sigma_{X}^{2}\left(1-\alpha_{2}^{2}\right)}}+\frac{\alpha_{2} x}{\sigma_{X}^{2}} e^{-\frac{x^{2}}{2 \sigma_{X}^{2}} \Phi}\left(\frac{\alpha_{2} x}{\sigma_{X} \sqrt{1-\alpha_{2}^{2}}}\right)
$$

where $\Phi(\quad)$ is the CDF of standard Gaussian distribution; and $p_{v}(x)=p_{u}(-x)$ can be easily derived since the valley is symmetrical with the peak.

On the other hand, the equivalent JPDF of peak and valley for $D_{R M}$ was derived as (Madsen et al. 1986; Tovo 2002)

$$
h_{R M}(u, v)=\frac{1}{\sigma_{X}^{2} \alpha_{2}^{2} 2 \sqrt{2 \pi}} \mathrm{e}^{-\frac{u^{2}+v^{2}}{4 \sigma_{X}^{2}\left(1-\alpha_{2}^{2}\right)}} \mathrm{e}^{-\frac{(u-v)^{2}\left(1-2 \alpha_{2}^{2}\right)}{4 \sigma_{X}^{2}\left(1-\alpha_{2}^{2}\right) 2 \alpha_{2}^{2}}}\left[\frac{u-v}{2 \sigma_{X} \sqrt{\left(1-\alpha_{2}^{2}\right)}}\right]
$$

It is readily illustrated that $h_{L C}(u, v)$ leads to a Rayleigh-type distribution of amplitude as

$$
p_{L C}(s)=\alpha_{2} \frac{s}{\sigma_{X}^{2}} e^{-\frac{s^{2}}{2 \sigma_{X}^{2}}}
$$

and gives the fatigue damage rate as shown in Eq. (6). 
Also, $h_{R M}(u, v)$ gives

$$
p_{R M}(s)=\frac{s}{\sigma_{X}^{2} \alpha_{2}^{2}} e^{-\frac{s^{2}}{2 \sigma_{X}^{2} \alpha_{2}^{2}}}
$$

and the fatigue damage rate given in Eq. (7).

\section{Spectral method for fatigue analysis of non-Gaussian processes}

\subsection{Non-Gaussian fatigue analysis based on translation process theory}

A non-Gaussian process $Z(t)$ with zero mean and unit STD can be related to an underlying standard Gaussian process $X(t)$ through a memoryless monotonic translation function (Grigoriu 1984 and 1995)

$$
z=g(x)=F_{Z}^{-1}[\Phi(x)]
$$

where $z$ and $x$ are standard non-Gaussian and Gaussian values; $g(\quad)$ is translation function; $F_{Z}$ and $\Phi$ are cumulative distribution functions (CDFs) of $Z(t)$ and $X(t) ; F_{Z}^{-1}$ is the inverse function of $F_{Z}$. A nonGaussian process with non-zero mean $\mu_{Z}$ and STD $\sigma_{Z}$ can always be normalized as a standard process by $\left(Z-\mu_{Z}\right) / \sigma_{Z}$. Practically, the translation function can be determined from mapping of CDFs, or represented by analytical translation models such as Hermite model (Wenterstein 1988).

From the translation process theory, the peak and valley in the underlying Gaussian process should be coincident with those of the non-Gaussian process due to the monotonic property of translation function, i.e., $z_{u}=\mu_{Z}+\sigma_{z} g\left(x_{u}\right)$ and $z_{v}=\mu_{Z}+\sigma_{Z} g\left(x_{v}\right)$, where $z_{u}$ and $z_{v}, x_{u}$ and $x_{v}$ are peak and valley of the non-Gaussian and underlying Gaussian processes. The amplitude and mean in the nonGaussian process are expressed as

$$
\begin{gathered}
s=\frac{1}{2}\left(z_{u}-z_{v}\right)=\frac{\sigma_{Z}}{2}\left[g\left(x_{u}\right)-g\left(x_{v}\right)\right] \\
m=\frac{1}{2}\left(z_{u}+z_{v}\right)=\mu_{z}+\frac{\sigma_{Z}}{2}\left[g\left(x_{u}\right)+g\left(x_{v}\right)\right]
\end{gathered}
$$

and their JPDF is given as follows due to the conservation of probability

$$
f_{R F}^{N G}(s, m) d s d m=h_{R F}^{G}\left(x_{u}, x_{v}\right) d x_{u} d x_{v}
$$

where $h_{R F}^{G}\left(x_{u}, x_{v}\right)$ is the JPDF of peak and valley in the underlying Gaussian process, which is given as 


$$
h_{R F}^{G}\left(x_{u}, x_{v}\right)=b h_{L C}^{G}\left(x_{u}, x_{v}\right)+(1-b) h_{R M}^{G}\left(x_{u}, x_{v}\right)
$$

where $h_{L C}^{G}\left(x_{p}, x_{v}\right)$ and $h_{R M}^{G}\left(x_{u}, x_{v}\right)$ are determined by Eqs. (17) and (19), respectively, both with a unit STD, i.e., $\sigma_{X}=1$.

Accordingly, the expected damage rate can be evaluated as

$$
E\left[D_{R F}^{n G}\right]=v_{p} C^{-1} \int_{0}^{\infty} s^{k} \int_{-\infty}^{\infty} f_{R F}^{N G}(s, m) d m d s=b E\left[D_{L C}^{n G}\right]+(1-b) E\left[D_{R M}^{n G}\right]
$$

where

$$
\begin{array}{r}
E\left[D_{L C}^{n G}\right]=v_{p} C^{-1}\left(\sigma_{Z} / 2\right)^{k} \int_{-\infty}^{\infty} \int_{-\infty}^{\infty}\left[g\left(x_{u}\right)-g\left(x_{v}\right)\right]^{k} h_{L C}^{G}\left(x_{u}, x_{v}\right) d x_{u} d x_{v} \\
=v_{p} C^{-1}\left(\sigma_{Z} / 2\right)^{k} \int_{-\infty}^{\infty}\left[g\left(x_{u}\right)-g\left(-x_{u}\right)\right]^{k} \alpha_{2} x_{u} \exp \left(-x_{u}^{2} / 2\right) d x_{u} \\
E\left[D_{R M}^{n G}\right]=v_{p} C^{-1}\left(\sigma_{Z} / 2\right)^{k} \int_{-\infty}^{\infty} \int_{-\infty}^{\infty}\left[g\left(x_{u}\right)-g\left(x_{v}\right)\right]^{k} h_{R M}^{G}\left(x_{u}, x_{v}\right) d x_{u} d x_{v}
\end{array}
$$

Following this derivation, the fatigue damage of a broad-band non-Gaussian process can be estimated as a linear combination of fatigue damages caused by level-crossing counting (narrow-band approximation) and range-mean counting methods, which is similar to the case of Gaussian process.

\subsection{Representation of non-Gaussian processes}

In general, a random process $Z(t)$ can be represented in terms of another random process $X(t)$ through orthogonal expansion. When $X(t)$ is a standard Gaussian process, the following Hermite translation model can be used in representing non-Gaussian processes, in which only the first four terms are included (Winterstein 1988)

$$
z=g(x)=\kappa\left[x+h_{3}\left(x^{2}-1\right)+h_{4}\left(x^{3}-3 x\right)\right]
$$

where $\kappa, h_{3}$ and $h_{4}$ are model coefficients.

For a softening non-Gaussian process with kurtosis in the range of $[3,15]$, the following semiempirical expressions were developed based on numerical calculations to minimize the difference of target skewness and kurtosis with those from the Hermite model (Winterstein and Kashef 2000): 


$$
\begin{aligned}
& \kappa=\frac{1}{\sqrt{1+2 h_{3}^{2}+6 h_{4}^{2}}} \\
& h_{3}=\frac{\gamma_{3}}{6}\left[\frac{1-0.015\left|\gamma_{3}\right|+0.3 \gamma_{3}^{2}}{1+0.2\left(\gamma_{4}-3\right)}\right] \\
& h_{4}=h_{40}\left[1-\frac{1.43 \gamma_{3}^{2}}{\gamma_{4}-3}\right]^{1-0.1\left(\gamma_{4}\right)^{0.8}} \\
& h_{40}=\frac{\left[1+1.25\left(\gamma_{4}-3\right)\right]^{1 / 3}-1}{10}
\end{aligned}
$$

where $\gamma_{3}$ and $\gamma_{4}$ are skewness and kurtosis, respectively.

The above monotonic Hermite model cannot be applied to a hardening non-Gaussian process with kurtosis less than 3 (Winterstein 1988). Intuitively, the Gaussian process may be represented in terms of Hermite polynomials of hardening non-Gaussian process as (e.g., Winterstein 1988)

$$
x=z-h_{3}\left(z^{2}-1\right)-h_{4}\left(z^{3}-3 z\right)
$$

However, by taking the expectation of both sides of above model, it is readily observed that this translation function cannot give zero mean of a Gaussian process when the non-Gaussian process is skewed. Therefore, a more general form of the translation function proposed in Ding and Chen (2015) for hardening non-Gaussian processes is adopted

$$
x=g^{-1}(z)=b_{2} z+b_{3}\left(z^{2}-\gamma_{3} z-1\right)+b_{4}\left(z^{3}-\gamma_{4} z-\gamma_{3}\right)
$$

where $b_{2}, b_{3}, b_{4}$ are model coefficients. It returns to the Hermite model when the expansion is on a Gaussian process with $\gamma_{3}=0$ and $\gamma_{4}=3$. Semi-empirical formulations of model coefficients $\left(b_{2}, b_{3}, b_{4}\right)$ in terms of skewness and kurtosis are established as (Ding and Chen 2015) 


$$
\begin{aligned}
& b_{2}=\varphi\left[1-\frac{\gamma_{3}^{4}+1.2 \gamma_{3}^{2}-0.18}{7.5 \exp \left(0.5 \gamma_{4}\right)}\right] \\
& b_{3}=-\frac{0.8 \gamma_{3}^{5}+\gamma_{3}^{3}+0.77 \gamma_{3}}{\left(\gamma_{4}-1\right)^{2}+0.5} \\
& b_{4}=-\varphi\left[0.04-\frac{11.5 \gamma_{3}^{4}+6.8 \gamma_{3}^{2}+3.5}{\left(\gamma_{4}^{2}+0.4\right)^{2}+0.15}\right] \\
& \varphi=\left[1-0.06\left(3-\gamma_{4}\right)\right]^{1 / 3}
\end{aligned}
$$

The corresponding translation function from Gaussian to non-Gaussian variables is then determined as

$$
z=g(x)=\left[\sqrt{\xi^{2}(x)+c}+\xi(x)\right]^{1 / 3}-\left[\sqrt{\xi^{2}(x)+c}-\xi(x)\right]^{1 / 3}-a
$$

where

$$
\begin{aligned}
& \xi(x)=\frac{x}{2 b_{4}}+\frac{\gamma_{3}}{2}+a\left(1.5+1.5 b-a^{2}\right) \\
& a=b_{3} /\left(3 b_{4}\right) ; b=\left(b_{2}-b_{3} \gamma_{3}-b_{4} \gamma_{4}\right) /\left(3 b_{4}\right) ; c=\left(b-a^{2}\right)^{3} .
\end{aligned}
$$

The application of moment-based translation model are limited to certain combination of process skewness and kurtosis, due to the monotonic requirement of translation function (Winterstein and MacKenzie 2012). Furthermore, it has been demonstrated in Ding and Chen (2014) that some nonGaussian processes cannot be well represented by a moment-based translation model. For instance of a process with bimodal distribution, the process kurtosis is no longer an indicator for its distribution tail. The moment-based translation model thus may give inaccurate representation of non-Gaussian process. In this case, a direct curve-fitting to a numerical translation function determined from CDF mapping leads to more accurate representation (Ding and Chen 2014). Adequate modeling of translation functions for various non-Gaussain processes will also be addressed later in the context of non-Gaussian fatigue evaluation. 


\section{Fatigue damage evaluation of Gaussian wind load effects}

\subsection{Alongwind response of a wind-excited tall building}

The effectiveness of TB spectral method for fatigue analysis of wind-excited tall buildings is examined. The building considered is an isolated tall building in urban environment with a rectangular cross section. The building height is $H=200 \mathrm{~m}$, and width is $B=0.2 H$. The fundamental frequencies in alongwind and crosswind directions are 0.230 and $0.276 \mathrm{~Hz}$, respectively. The fundamental modal shapes in these two directions are assumed to be linear over the building height. The generalized force $Q(t)$ is related to the base bending moment $M(t)$ as $Q(t)=M(t) / H$, while $M(t)$ can be determined in wind tunnels from high frequency force balance (HFFB) experiments or integral of wind pressure measurements (e.g., Chen and Kareem 2005a; Lin et al. 2005). In this study, the PSD of alongwind base bending moment coefficient $C_{M}(t)$ suggested in Architectural Institute of Japan (AIJ) recommendations (AIJ 2004) is used, which is summarized in Appendix A.

The PSD and root-mean-square (RMS) value of the building top displacement are evaluated from the PSD of generalized force and the properties of the structural system. The base bending moment response is then calculated by multiplying $M_{1}\left(2 \pi f_{1}\right)^{2} H$, where $M_{1}$ is the generalized mass of the fundamental mode, and $M_{1}=m H / 3 ; m$ is building mass per unit height; and $f_{1}$ is the fundamental frequency. The stress response of interest is calculated from the base bending moment response by multiplying a constant $\varphi_{s}$, which represents the stress due to unit base bending moment. Fig. 1(a) shows the ratio of RMS resonant and background (quasi-static) base bending stresses, denoted as $\sigma_{X r}$ and $\sigma_{X b}$, respectively, as a function of wind speed and modal damping ratio $\zeta$. The bandwidth parameters $\alpha_{1}$ and $\alpha_{2}$ calculated from response PSD function are portrayed in Fig. 1(b). With the increasing wind speed, the resonant response becomes more dominant, and the response process becomes more narrow-banded, especially for a smaller modal damping ratio. Since only the fundamental modal response is considered, the building top displacement has the same features as that of building base bending moment. Closed-form formulation 
can be also used for calculating the spectral moments, mean crossing rate and bandwidth parameters of the displacement (Appendix B).

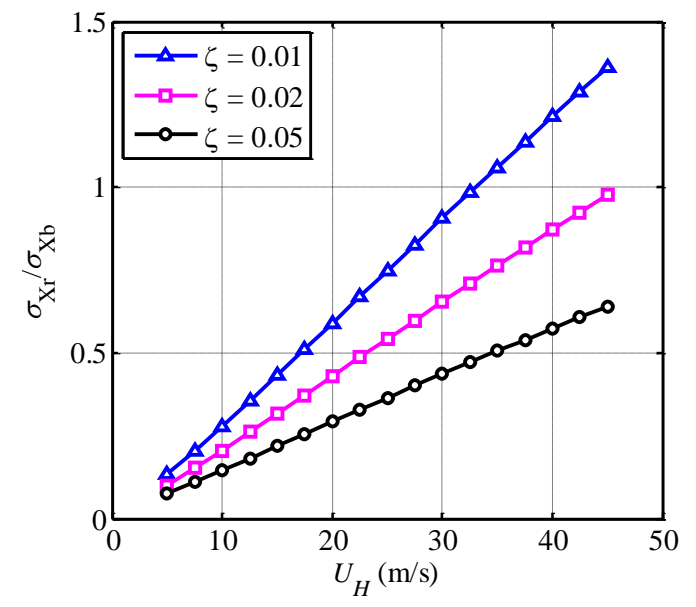

(a) Ratio of resonant and background responses
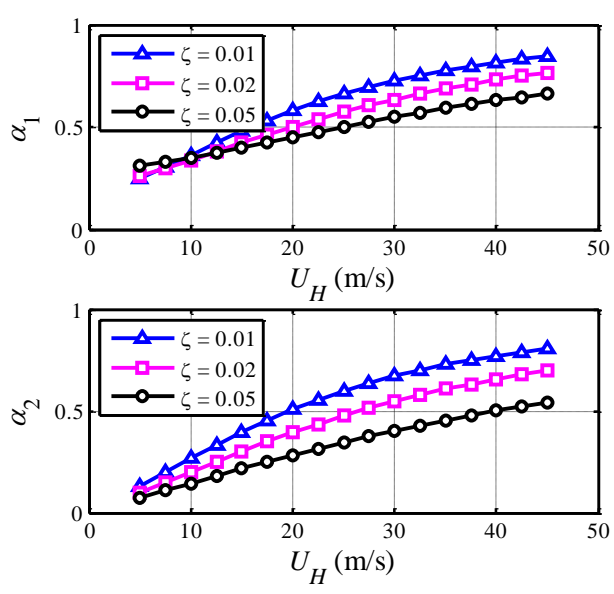

(b) Bandwidth parameters $\alpha_{1}$ and $\alpha_{2}$

Fig. 1 Characteristics of alongwind response

Fig. 2(a) shows the $b$ value of TB spectral method calculated from bandwidth parameters $\alpha_{1}$ and $\alpha_{2}$. Fig. 2(b) portrays the fatigue damage rate calculated from TB spectral method, denoted as $E\left[D_{T B}\right]$ and its upper and lower bounds from level-crossing counting method $\left(E\left[D_{L C}\right]\right)$ and range-mean counting method $\left(E\left[D_{R M}\right]\right)$, where $E\left[D_{L C}\right]$ and $E\left[D_{R M}\right]$ are calculated from the closed-form expression as shown in Eqs. (6) and (7). Only the results for damping ratio $\zeta=0.02$ are given for illustration. The fatigue damage rate is normalized by $\varphi_{S}^{k} U_{H}^{2 k} / C$, where $U_{H}$ is the mean wind speed at building height; the exponent of S-N curve is selected as $k=3$. As the fatigue damage rate shown in Fig. $2(\mathrm{~b})$ is normalized by $U_{H}^{2 k}$, it increases much faster than what displayed with the increase of mean wind speed.

To examine the accuracy of estimations from TB spectral method, rainflow counting damage rate $E\left[D_{R F}\right]$ is also calculated using simulated response time histories. The generalized force time history is simulated following the spectral representation method (Shinozuka and Jan 1972; Chen and Kareem 2005b). The response time history is then calculated by Newmark's step-by-step method. In order to achieve a reliable estimation of fatigue damage, 10000 short-term time histories are generated, each with time duration of $10 \mathrm{~min}$ and sampling frequency of $10 \mathrm{~Hz}$. The comparison of both estimates is shown in 
Fig. 2(c). The results illustrate that the prediction from TB spectral method is satisfactory and the associated relative error is less than $5 \%$.

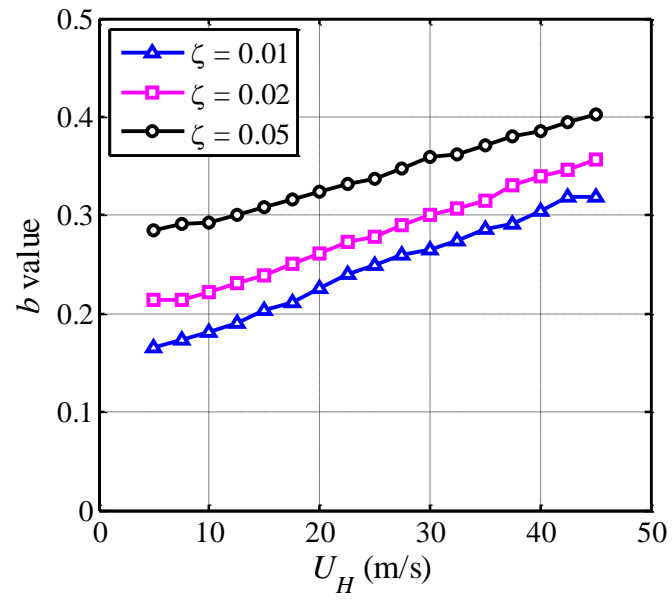

(a) $b$ value of TB spectral method

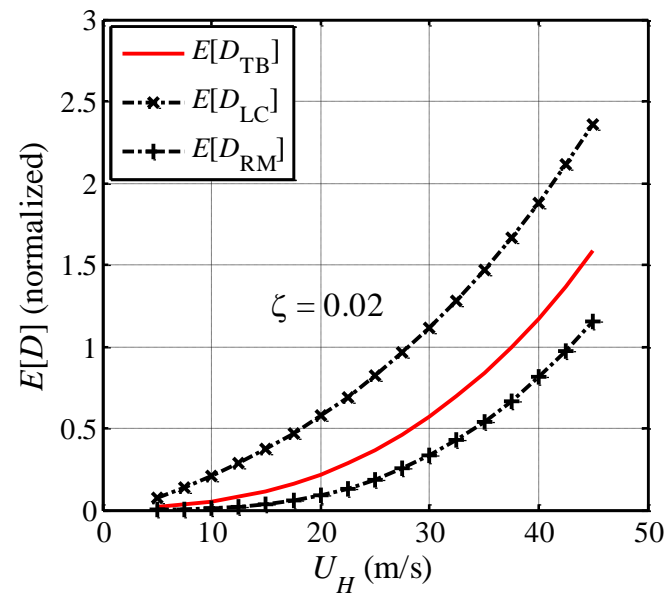

(b) $E\left[D_{T B}\right]$ and its two bounds $E\left[D_{L C}\right]$ and $E\left[D_{R M}\right]$

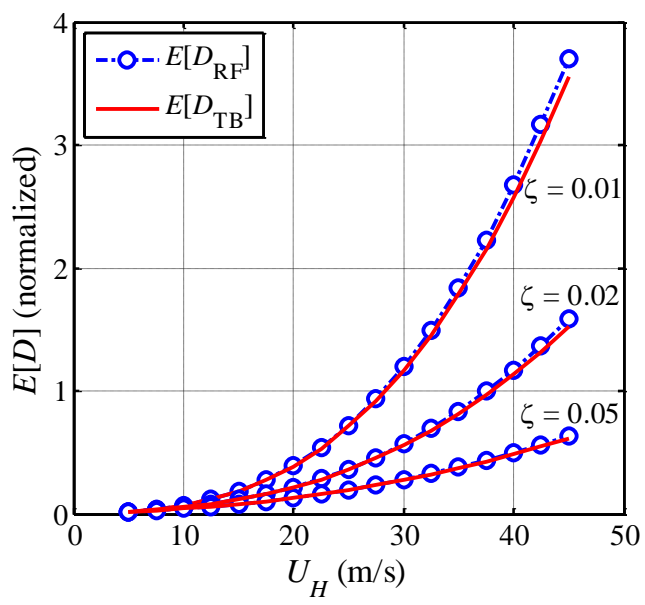

(c) $E\left[D_{T B}\right]$ and $E\left[D_{R F}\right]$

Fig. 2 Comparison of fatigue damage rate by rainflow counting and spectral methods (alongwind response)

\subsection{Crosswind response of a wind-excited tall building}

Similar calculations for crosswind-induced fatigue damage of the building are also performed based on the PSD of base bending moment coefficient introduced in Appendix A. The ratio of RMS resonant 
and background base bending stress is calculated from its PSD function and displayed in Fig. 3(a). The bandwidth parameters $\alpha_{1}$ and $\alpha_{2}$ are given in Fig. 3(b). It is observed that the resonant component in crosswind response is more significant than in the alongwind response and the crosswind response process is more narrow-banded. Fig. 4(a) portrays the $b$ value of TB spectral method. Fig. 4(b) shows the estimated fatigue damage rate $E\left[D_{T B}\right]$ and its upper and lower bounds $E\left[D_{L C}\right]$ and $E\left[D_{R M}\right]$, which are also normalized values as did for anlongwind response. In this case, the difference between two bounds is smaller than the alongwind case since the stress process is more narrow-banded. It is also observed that the fatigue damage caused by crosswind response is more significant. Figs. 4(c) shows the comparison between normalized $E\left[D_{T B}\right]$ and $E\left[D_{R F}\right]$, which agrees well with each other. The prediction error is very small and the performance of TB spectral method is satisfactory.

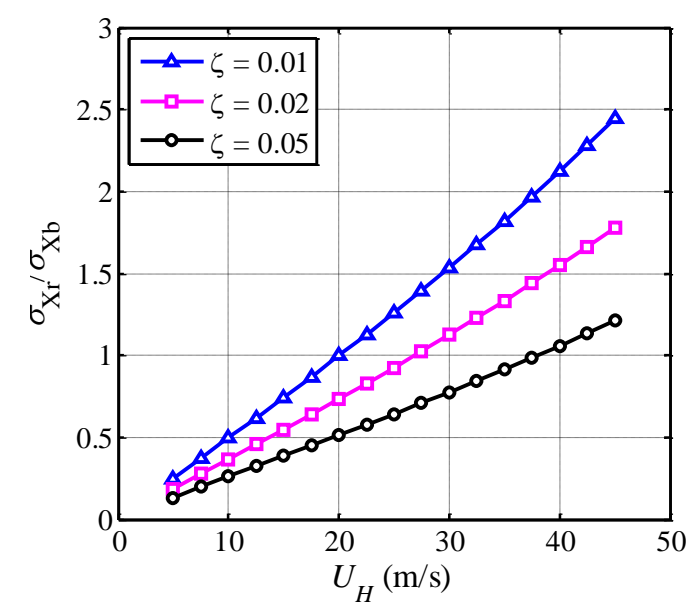

(a) Ratio of resonant and background responses
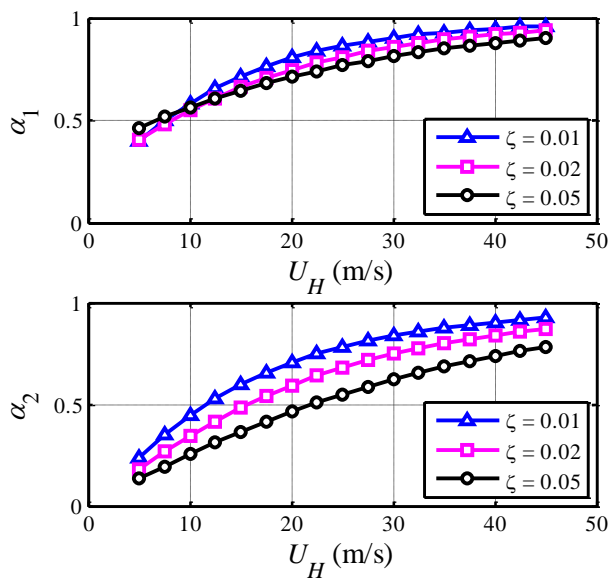

(b) Bandwidth parameters $\alpha_{1}$ and $\alpha_{2}$

Fig. 3 Characteristics of crosswind response 


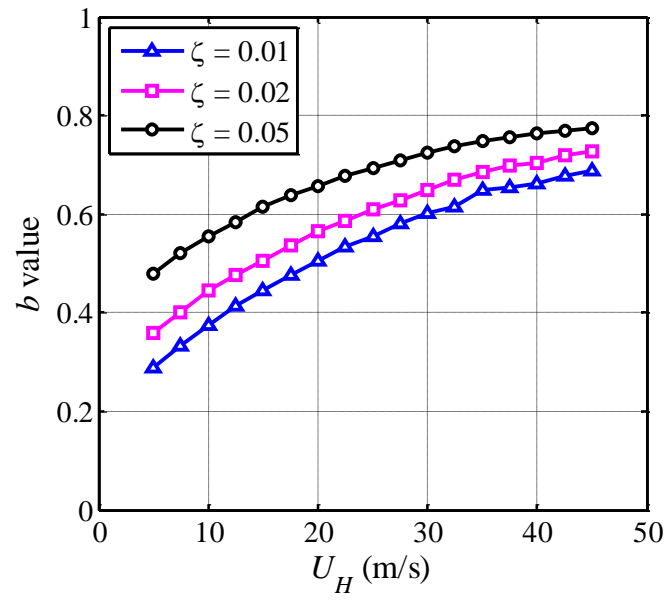

(a) $b$ value of TB spectral method

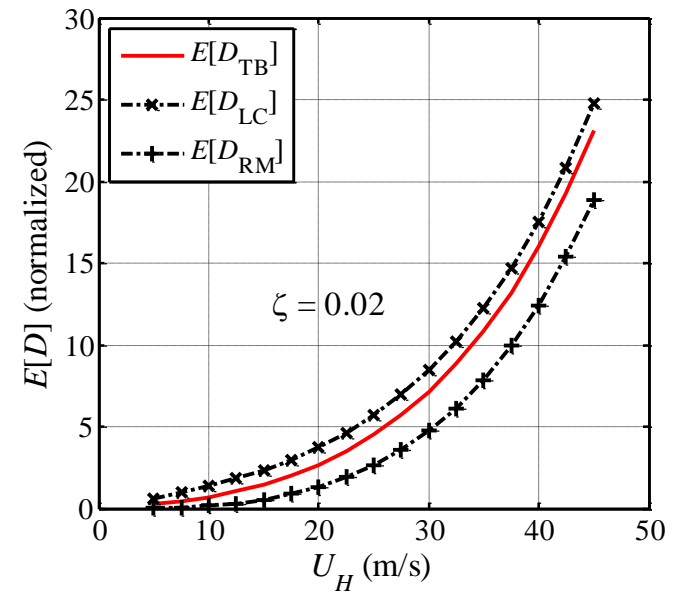

(b) $E\left[D_{T B}\right]$ and its two bounds $E\left[D_{L C}\right]$ and $E\left[D_{R M}\right]$

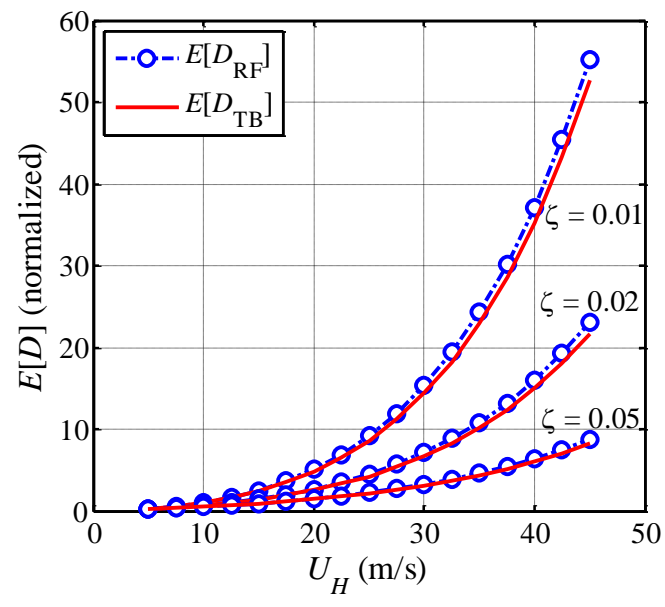

(c) $E\left[D_{T B}\right]$ and $E\left[D_{R F}\right]$

Fig. 4 Comparison of fatigue damage rate by rainflow counting and spectral methods (crosswind response)

\subsection{Coupled alongwind and crosswind response of a wind-excited tall building}

The stress of a column at building base is contributed by bending normal stresses in both alongwind and crosswind directions. In the following, the coupled response as the sum of alongwind and crosswind base bending moments is considered. Figs. 5(a) and (b) show the ratio of RMS alongwind to crosswind base bending stresses, denoted as $\sigma_{X a}$ and $\sigma_{X c}$, and the bandwidth parameters $\alpha_{1}$ and $\alpha_{2}$. Figs. 6(a), (b) and (c) portray the $b$ value of TB spectral method, the normalized $E\left[D_{T B}\right]$ and its upper and lower bounds, and the comparison of $E\left[D_{T B}\right]$ and $E\left[D_{R F}\right]$. The prediction error is small and the performance of 
TB spectral method is also satisfactory. It is evident that the fatigue damage of this coupled response is dominated by the contribution of crosswind response. Studies for buildings with different alongwind and crosswind modal frequencies were also performed, and similar performance of the TB spectral method was observed but not displayed for the sake of simplicity.

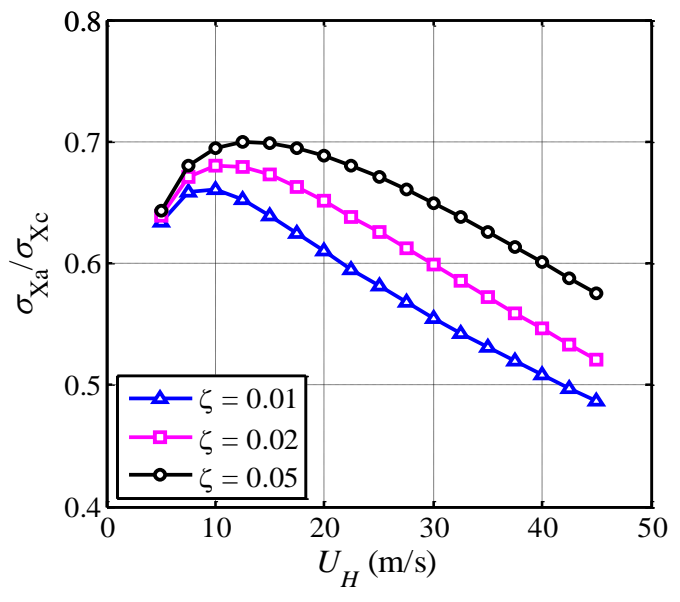

(a) Ratio of alongwind and crosswind responses
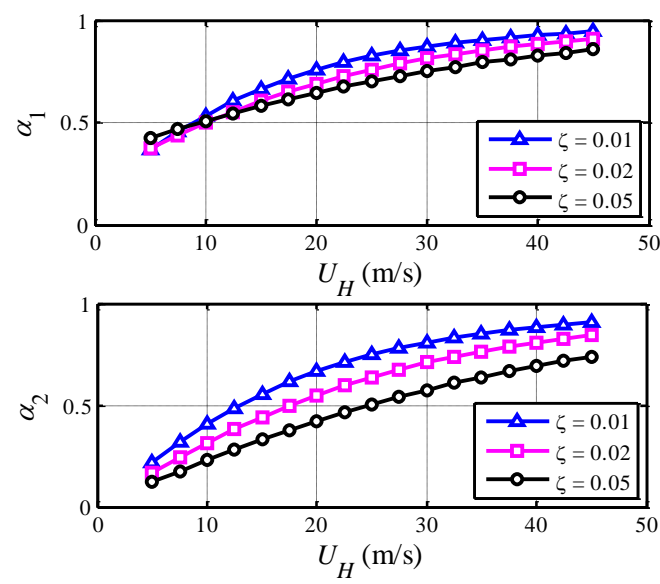

(b) Bandwidth parameters $\alpha_{1}$ and $\alpha_{2}$

Fig. 5 Characteristics of coupled alongwind and crosswind response

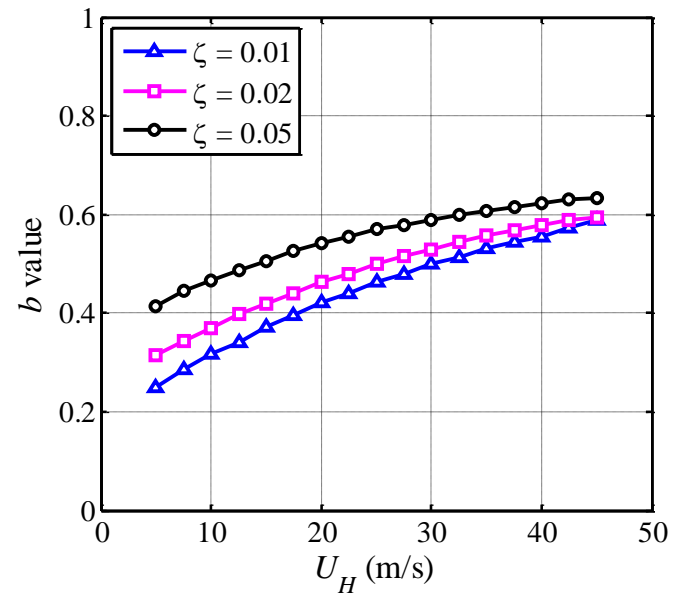

(a) $b$ value of TB spectral method

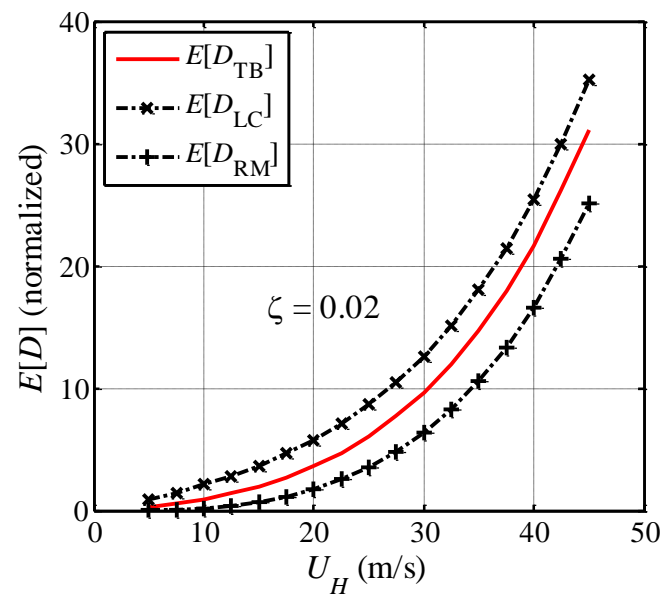

(b) $E\left[D_{T B}\right]$ and its two bounds $E\left[D_{L C}\right]$ and $E\left[D_{R M}\right]$ 


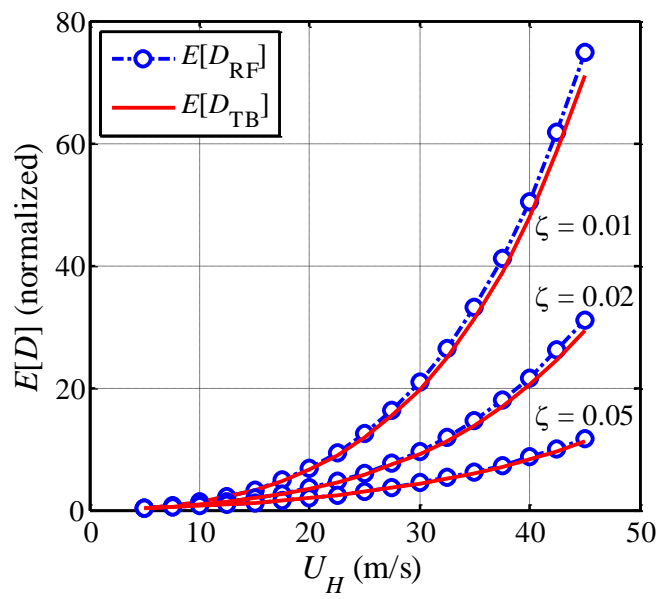

(c) $E\left[D_{T B}\right]$ and $E\left[D_{R F}\right]$

Fig. 6 Comparison of fatigue damage rate by rainflow counting and spectral methods (coupled response)

\subsection{Wind pressures on claddings as broad-band Gaussian processes}

For cladding responses, the dynamic effects of time varying excitation can be neglected, i.e., the resonant response is neglected and only the background response is considered. The properties of the stochastic stress process are represented by those of the wind pressure process (excitation), including the power spectrum and probability distribution. Fig. 7 shows three typical PSD functions of broad-band Gaussian wind pressure coefficient processes on a saddle-shaped large-span roof (Ding and Chen 2014). The sampling frequency is $6.25 \mathrm{~Hz}$ in prototype scale. The PSD can be represented in the following general form

$$
\frac{f S(f)}{\sigma^{2}}=\frac{\kappa f}{\left(1+\beta_{1} f^{\beta_{2}}\right)^{\beta_{3}}}
$$

in which $\kappa, \beta_{1}, \beta_{2}, \beta_{3}$ are model coefficients. Table 1 lists the model coefficients for these three processes, while the performance of this model is demonstrated in Fig. 7. 


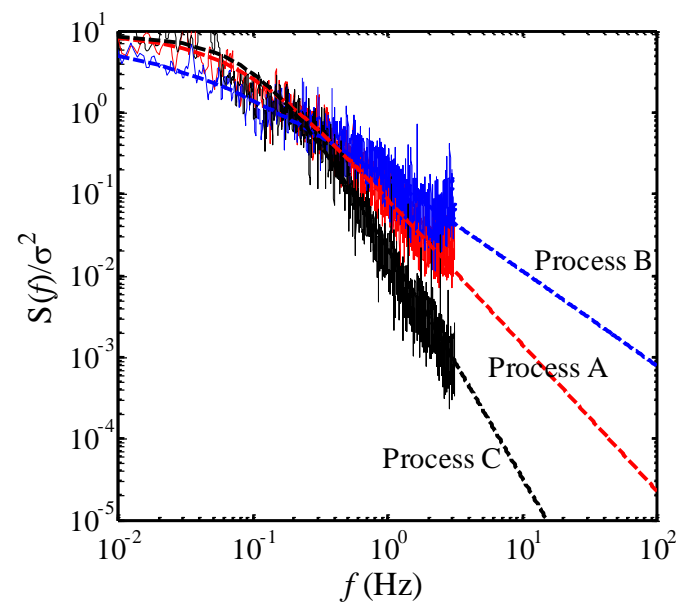

Fig. 7 PSDs of selected wind pressure coefficients processes

In the following, the fatigue damage evaluation is addressed using pressure coefficient process which relates to cladding stress by a constant $\varphi_{c}$. The fatigue damages associated are calculated from both TB spectral method and rainflow counting method. The results are also listed in Table 1, where the fatigue damage rate is normalized by $\varphi_{c}^{k} / C$. The relative error shown is the estimation from TB spectral method $\left(E\left[D_{T B}\right]\right)$ with respect to that from rainflow counting method $\left(E\left[D_{R F}\right]\right)$. It is clear that the prediction from TB spectral method with $b$ value estimated from Eq. (11) gives a noticeable error.

Table 1 Normalized fatigue damage rate and relative error by TB spectral method

\begin{tabular}{|c|c|c|c|c|c|c|c|c|c|}
\hline \multirow{2}{*}{ Process } & \multicolumn{4}{|c|}{ Model coefficients } & \multicolumn{2}{|c|}{$\begin{array}{l}\text { Bandwidth } \\
\text { parameters }\end{array}$} & \multirow{2}{*}{$E\left[D_{R F}\right]$} & \multirow{2}{*}{$\begin{array}{c}E\left[D_{T B}\right] \\
(b)\end{array}$} & \multirow{2}{*}{$\begin{array}{c}E\left[D_{T B}\right] \\
\left(b_{\text {new }}\right)\end{array}$} \\
\hline & $\kappa$ & $\beta_{1}$ & $\beta_{2}$ & $\beta_{3}$ & $\alpha_{1}$ & $\alpha_{2}$ & & & \\
\hline A & 9.04 & 24.1 & 1.24 & 1.45 & 0.51 & 0.28 & 0.84 & $0.96(14 \%)$ & $0.87(3 \%)$ \\
\hline B & 9.89 & 11.1 & 0.73 & 1.61 & 0.62 & 0.44 & 1.59 & $1.84(16 \%)$ & $1.65(4 \%)$ \\
\hline $\mathrm{C}$ & 9.96 & 19.5 & 1.42 & 2.01 & 0.52 & 0.18 & 0.46 & $0.50(9 \%)$ & $0.47(3 \%)$ \\
\hline
\end{tabular}

\subsection{New formulation for the intermediate value $b$ of TB spectral method}

To achieve a better prediction of wind-induced fatigue damage using TB spectral method, an improved calculation of the intermediate value $b$ as a function of parameters $\alpha_{1}$ and $\alpha_{2}$ is explored. A wide range of bandwidth parameters $\alpha_{1}$ and $\alpha_{2}$ are covered by changing the model coefficients $\beta_{1}, \beta_{2}$ and 
$\beta_{3}$ of the PSD model given by Eq. (36). Fig. 8 shows the possible combinations of bandwidth parameters for the PSD model discussed, in which the results of previously discussed alongwind, crosswind and coupled building responses are also presented. For a given PSD with a specific combination of $\alpha_{1}$ and $\alpha_{2}$, a total of 10000 short-term $(10 \mathrm{~min})$ time histories is generated using spectral representation method with sampling frequency of $6.25 \mathrm{~Hz}$. The rainflow counting damage $E\left[D_{R F}\right]$ is then calculated and used to determine the required $b$ value. It is followed by a regression to a functional form in terms of $\alpha_{1}$ and $\alpha_{2}$, which yields the following empirical expression

$$
b_{\text {new }}=\frac{\left(\alpha_{1}-\alpha_{2}\right)\left(1-\alpha_{1} \alpha_{2}\right)}{\left(1-\alpha_{2}\right)^{2}}
$$

It should be noted that the original expression for $b$ value, Eq. (11), introduced in TB spectral method was developed in the same procedure but covered a wider range of PSD shapes. The performance of the newly introduced formulation is demonstrated in Fig. 9, where only part of combinations of $\alpha_{1}$ and $\alpha_{2}$ is presented. The newly introduced formulation gives a better fit to the required parameter $b$. As shown in Tables 1, the TB spectral method with this newly introduced expression results in better prediction of fatige damage of broad-band Gaussian wind effects.

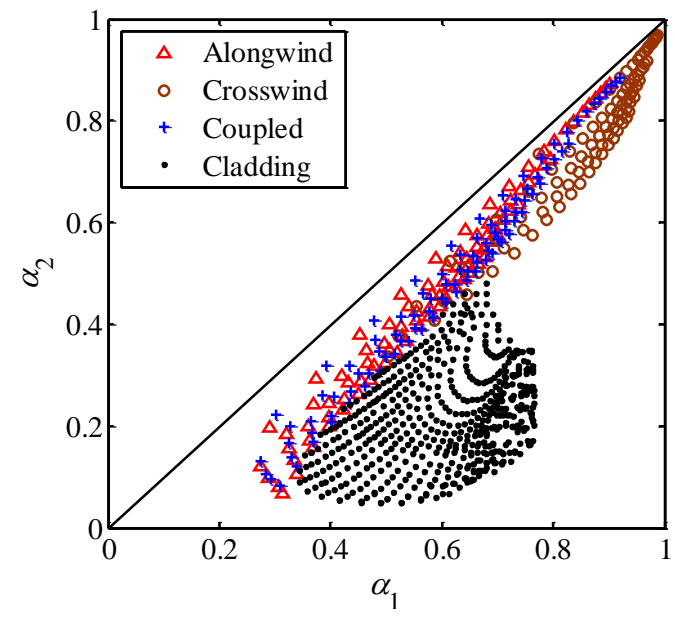

Fig. 8 Possible combinations of bandwidth parameters for different PSD models 


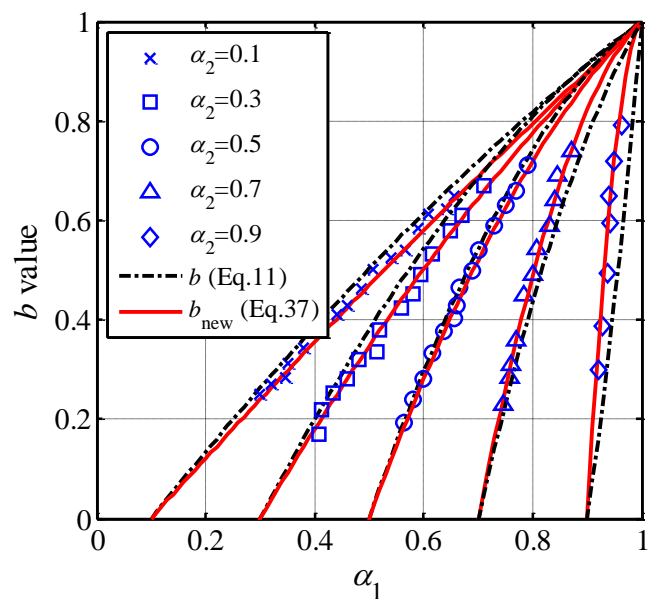

Fig. 9 Comparison of numerical and approximated $b$ value

\section{Fatigue damage evaluation of non-Gaussian wind load effects on claddings}

\subsection{Synthetic non-Gaussian processes with prescribed translation functions}

Full-scale and wind tunnel measurement data have shown that wind pressures on claddings may be of significant non-Gaussian characteristics with a variety of probability distributions (e.g., Holmes 1981; Yeatts and Mehta 1993; Kumar and Stathopoulos 1998). To validate the effectiveness of TB spectral method as applied to non-Gaussian fatigue damage evaluation, synthetic non-Gaussian processes with prescribed translation functions are first investigated. The translation function is determined from process skewness and kurtosis. The PSD function of the underlying Gaussian process is given as the model in Eq. (36) with $\kappa=9.04, \beta_{1}=24.1, \beta_{2}=1.24$, and $\beta_{3}=1.45$, i.e., $\alpha_{1}=0.51$ and $\alpha_{2}=0.28$, which is identical to that of process A addressed previously. The generation of non-Gaussian process samples is achieved by first generating underlying Gaussian process samples using the spectral representation method, and followed by a translation to non-Gaussian samples (e.g., Gong et al. 2014). The PSD functions and bandwidth parameters of different non-Gaussian processes featuring different skewness and kurtosis are almost the same. The rainflow counting damage is calculated and the corresponding $b$ value is determined such that the TB spectral method gives the target fatigue damage. Fig. 10(a) displays the required $b$ value as a function of skewness and kurtosis. The values calculated from analytical expressions 
defined by Eqs. (11) and (37), i.e., $b$ and $b_{\text {new }}$, are also given. Same examination is also carried out for processes with bimodal spectral shape with bandwidth parameters $\alpha_{1}=0.86$ and $\alpha_{2}=0.79$, which is resulted from coupled alongwind and crosswind response. The results are summarized in Fig. 10(b).

Based on these two examples, it is observed that the $b$ value is almost not influenced by the nonGaussian characteristics, which illustrates the influences of spectral properties and non-Gaussian characteristics on fatigue damage can be separated. It is also observed that the newly proposed expression leads to a better prediction.

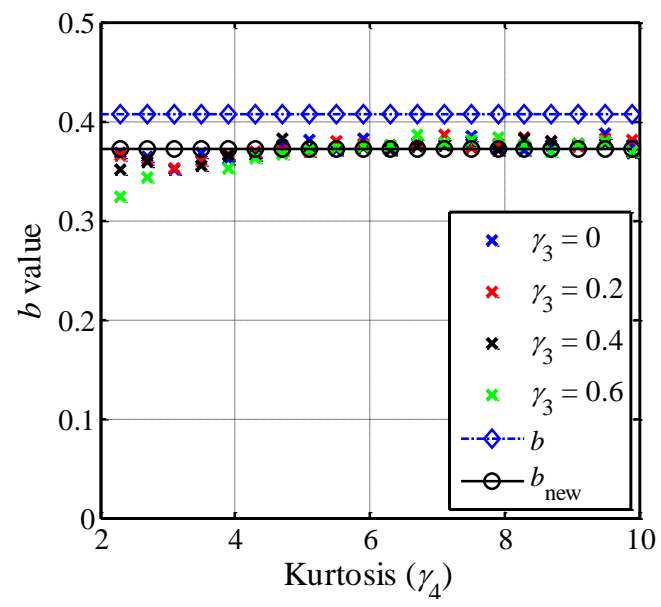

(a) $\alpha_{1}=0.51$ and $\alpha_{2}=0.28$

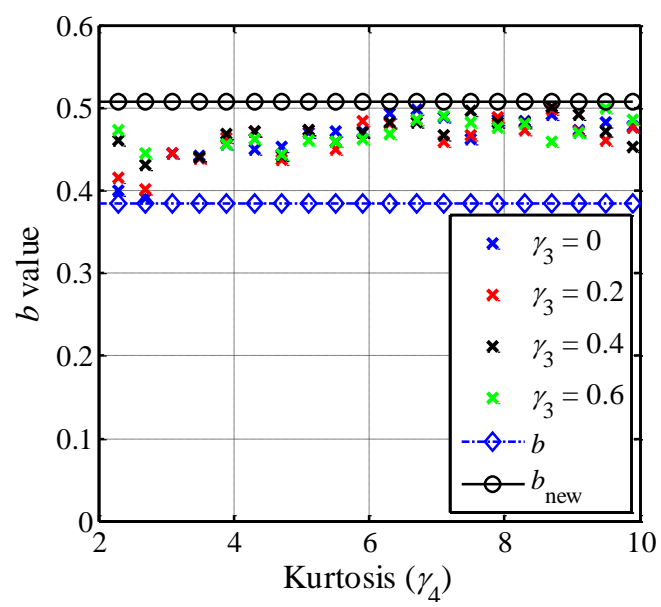

(b) $\alpha_{1}=0.86$ and $\alpha_{2}=0.79$

Fig. $10 b$ value for different non-Gaussian processes

For Gaussian processes, the TB spectral method can also be considered as a method to provide a correction factor of narrow-band approximation as Eq. (12). If this relationship is directly applied to fatigue damage evaluation of non-Gaussian processes, an approximation of the non-Gaussian fatigue is presented as

$$
E\left[D_{R F}^{n G}\right]=\left[b+(1-b) \alpha_{2}^{k-1}\right] E\left[D_{N B}^{n G}\right]
$$

Fig. 11 shows the results from this approximation as compared to TB spectral method and rainflow counting method for a series of non-Gaussian processes with zero skewness, while similar results are observed for skewed cases. It demonstrates that the estimation from non-Gaussian TB spectral method 
generally performs better than that from Eq. (38). The expression of $b_{\text {new }}$ is adopted in both calculations. Eqs. (26) and (38) are not identical in the case of non-Gaussian fatigue damage as the relation $E\left[D_{R M}^{n G}\right]=$ $\alpha_{2}^{k-1} E\left[D_{N B}^{n G}\right]$ cannot be derived for non-Gaussian processes.

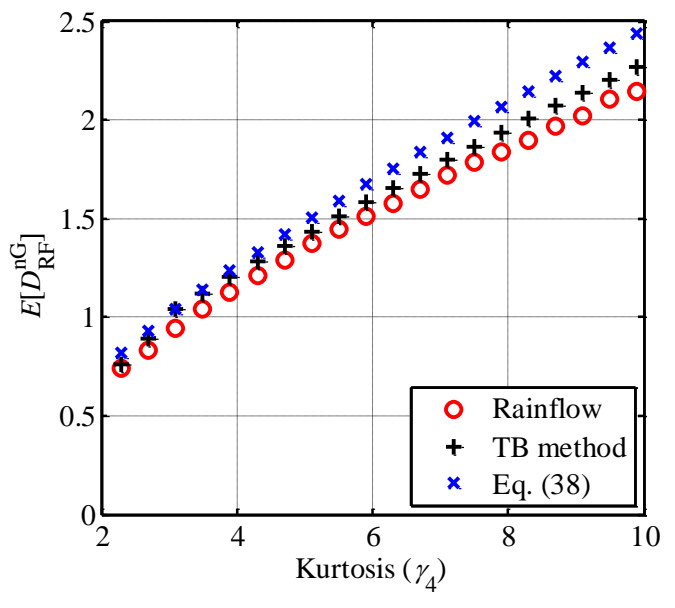

(a) $\alpha_{1}=0.51$ and $\alpha_{2}=0.28$

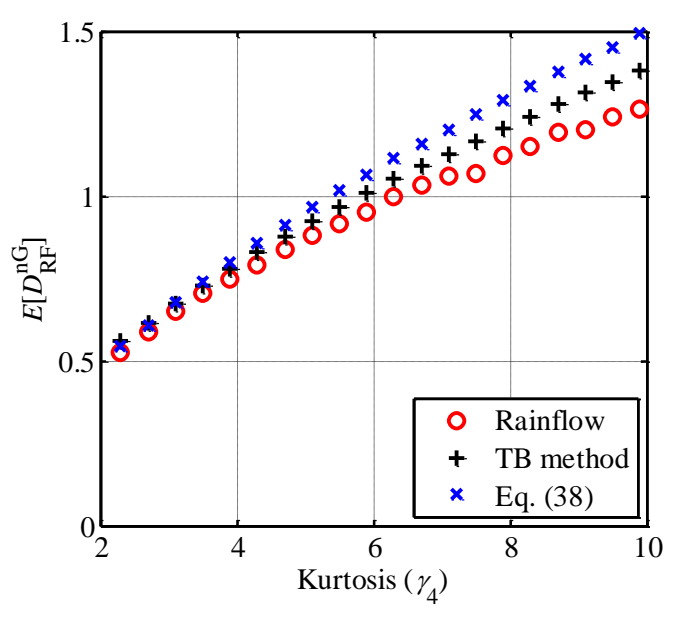

(b) $\alpha_{1}=0.86$ and $\alpha_{2}=0.79$

Fig. 11 Fatigue damage rate from TB spectral method with non-Gaussian consideration

\subsection{Experimental wind pressure coefficient data}

\subsubsection{Non-Gaussian distribution and broad-band spectral properties}

The wind pressure coefficient data on a saddle-shaped large-span roof were collected from wind tunnel test, which is conducted at Beijing Jiaotong University in China. The data collected consist 275 sets of wind pressure records each with time duration of $10 \mathrm{~min}$ and a positive value means suction pressure. In this application, wind pressures at 6 locations with different probability distributions are selected to examine the effectiveness of the spectral method introduced for non-Gaussian fatigue damage evaluation. The time history of different pressure coefficients are displayed in Fig. 12. The first four statistic moments of these pressure coefficients are shown in Table 2. The PSD and PDF of these processes are displayed in Figs. 13 and 14. The statistical moments, PSD and PDF are estimated via ensemble average of 275 samples. It is observed that the spectral properties for different wind pressure processes are very similar, although they have different probability distributions. The peak factor defined 
by mean extreme (subtracts the process mean) over process STD (Davenport 1964) is also shown in Table 2, which is aimed to demonstrate the significant influence of non-Gaussian effects on extremes. The peak factors associated with both maximum and minimum are presented.
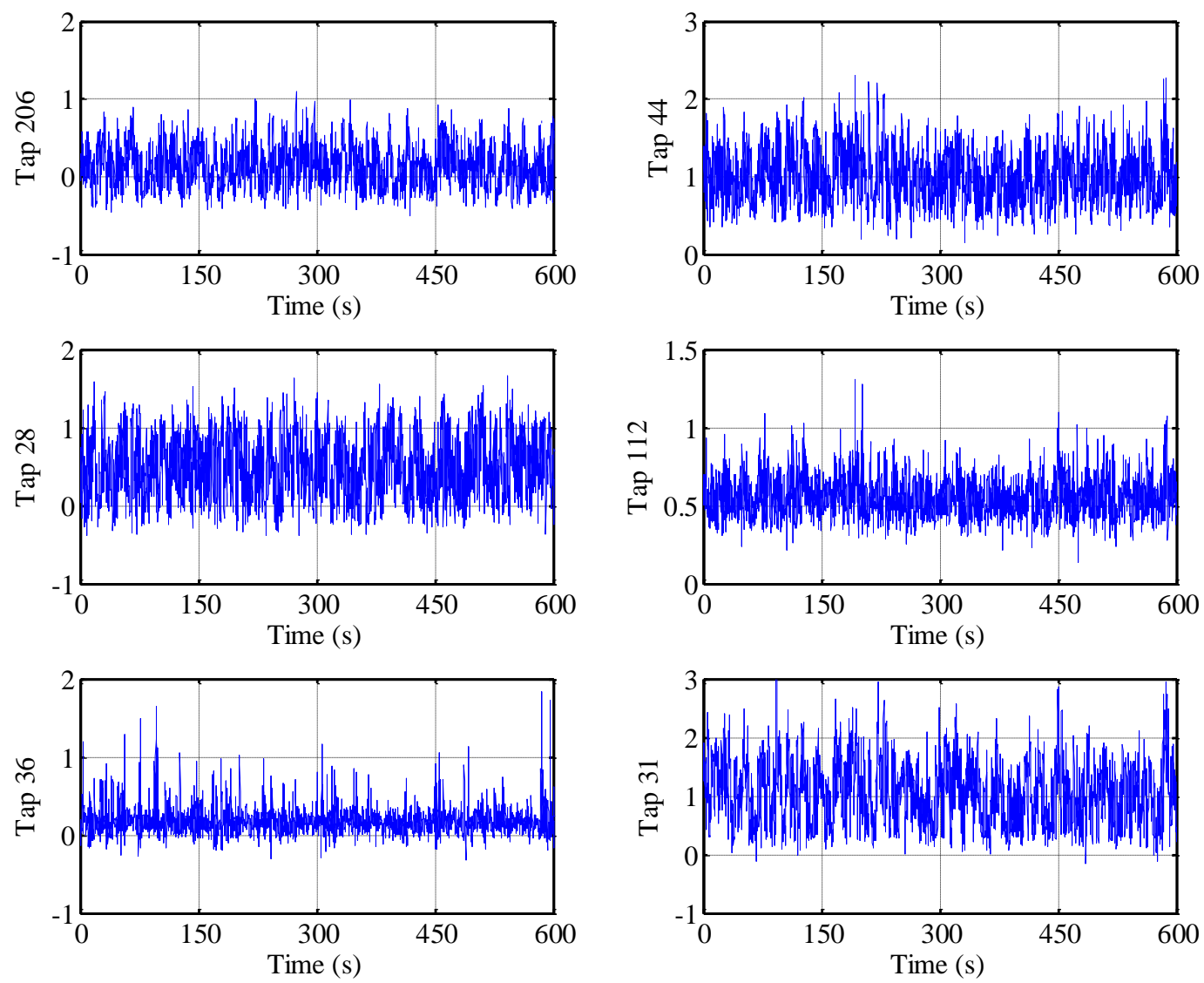

Fig. 12 Time history of pressure coefficients at different locations

Table 2 Statistics of pressure coefficients

\begin{tabular}{|c|c|c|c|c|c|c|c|}
\hline \multirow{3}{*}{ Tap No. } & \multirow{3}{*}{ Mean } & \multirow{3}{*}{ STD } & \multirow{3}{*}{$\begin{array}{c}\text { Skewness } \\
\left(\gamma_{3}\right)\end{array}$} & \multirow{3}{*}{$\begin{array}{l}\text { Kurtosis } \\
\qquad\left(\gamma_{4}\right)\end{array}$} & \multicolumn{3}{|c|}{ Peak factor $(T=10 \mathrm{~min})$} \\
\hline & & & & & \multicolumn{2}{|c|}{ Data } & \multirow{2}{*}{$\begin{array}{l}\text { Davenport's formula } \\
\text { (Gaussian assumption }\end{array}$} \\
\hline & & & & & Max. & Min. & \\
\hline Tap 206 & 0.14 & 0.24 & 0.10 & 3.05 & 4.06 & -3.74 & 3.84 \\
\hline Tap 44 & 1.01 & 0.32 & 0.42 & 3.04 & 4.17 & -2.74 & 3.82 \\
\hline Tap 28 & 0.51 & 0.37 & -0.20 & 2.65 & 3.02 & -3.68 & 3.84 \\
\hline Tap 112 & 0.55 & 0.13 & 0.70 & 4.35 & 5.56 & -3.21 & 3.86 \\
\hline Tap 36 & 0.19 & 0.16 & 2.22 & 14.7 & 9.18 & -3.53 & 3.85 \\
\hline Tap 31 & 1.06 & 0.49 & 0.22 & 2.50 & 3.81 & -2.34 & 3.85 \\
\hline
\end{tabular}




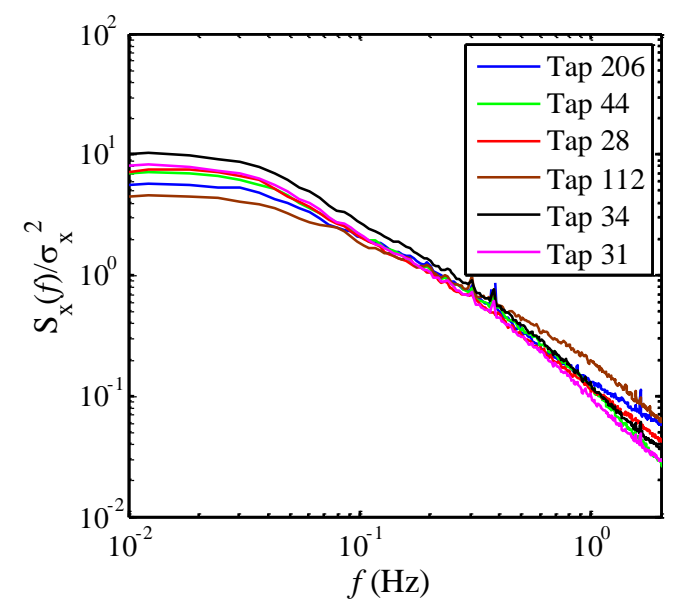

Fig. 13 PSDs of pressure coefficient processes
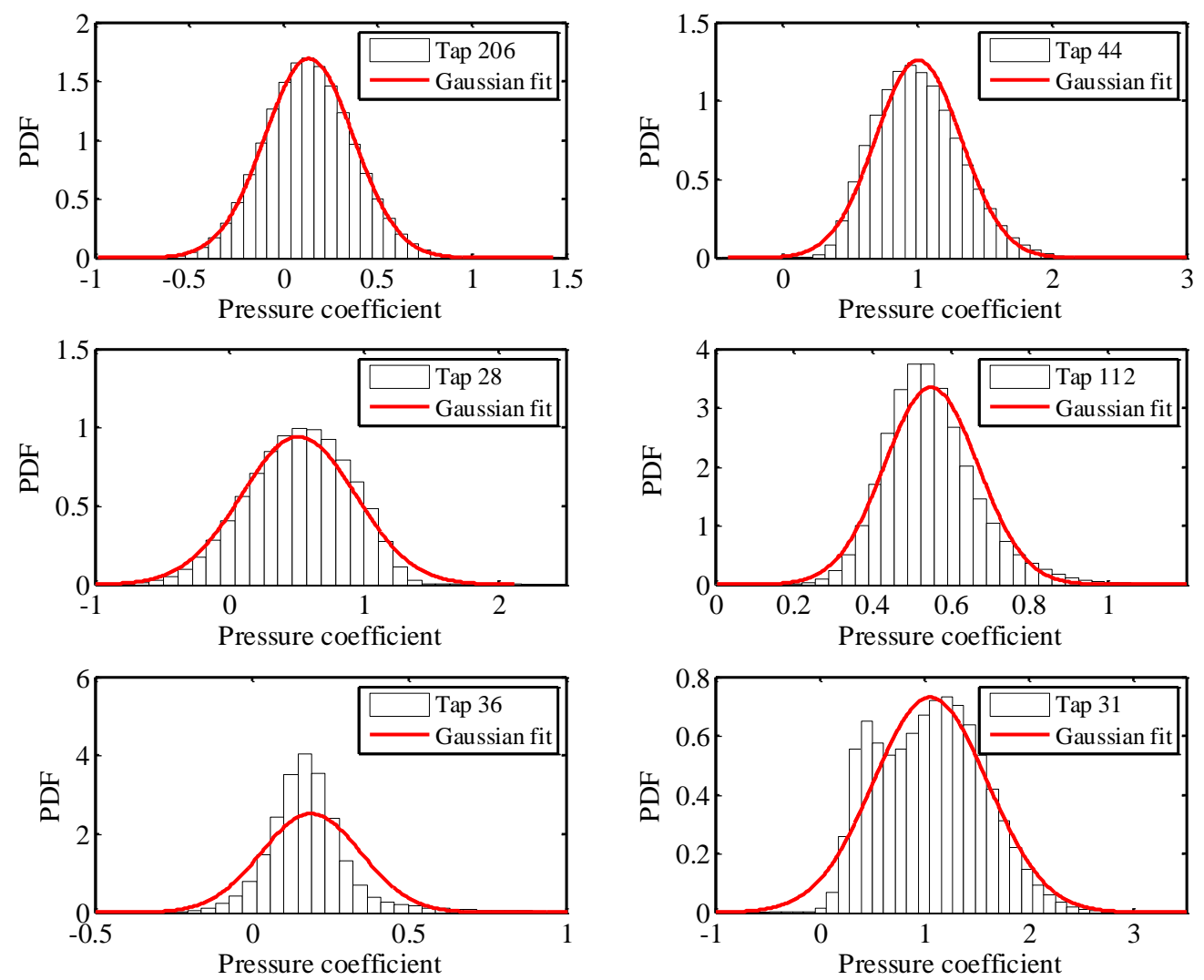

Fig. 14 PDFs of pressure coefficient processes in terms of histogram and Gaussian fit 


\subsubsection{Fatigue loading spectrum and fatigue damage from spectral method}

The bandwidth parameters $\alpha_{1}$ and $\alpha_{2}$, and the calculated $b$ value from different sample size is compared in order to demonstrate their uncertainties. The results from ensemble average of $n$ short-term (10 min) samples are denoted as $\alpha_{1}^{(n)}, \alpha_{2}^{(n)}, b^{(n)}$ and $n=5$ is displayed in Figs. 15 and 16, while the ensemble averaged values from all 275 short-term samples are denoted as $\alpha_{1}, \alpha_{2}, b$ and summarized in Table 3. The variations of bandwidth parameters and the $b$ value are quite small.

To calculate the fatigue damage of these non-Gaussian processes with TB spectral method, the analytical translation function models must be established first. Fig. 17 shows the numerical translation functions determined from data by mapping of CDFs, along with moment-based translation functions. For softening non-Gaussian processes, the Hermite model defined by Eq. (29) is applied, while for hardening non-Gaussian processes, the translation model defined by Eq. (32) is used. It is demonstrated that the moment-based translation model can well represent the mildly non-Gaussian processes of Taps 206, 28 and 112. The combination of skewness and kurtosis of the process of Tap 44 is beyond the application region of moment-based model, i.e., $3+\left(1.25 \gamma_{3}\right)^{2}>\gamma_{4}$ (Winterstein and MacKenzie 2012; Ding and Chen 2014), so the moment-based translation model is not applicable. For strongly non-Gaussian process of Tap 36, the translation model constructed from first four statistic moments is not accurate, especially for the negative tail. The process of Tap 31 follows a bimodal distribution, for which the kurtosis is no longer an indicator for its upper tail behaviors, thus the moment-based translation model is incorrect.

Table 3 Normalized fatigue damage rate from rainflow counting and spectral methods

\begin{tabular}{lccccccc}
\hline & & & & & \multicolumn{3}{c}{ TB spectral method $\left(E\left[D_{T B}\right]\right)$} \\
\cline { 6 - 7 } Processes & $E\left[D_{R F}\right]$ & $\alpha_{1}$ & $\alpha_{2}$ & $b_{\text {new }}$ & $\begin{array}{c}\text { Gaussian } \\
\text { assumption }\end{array}$ & $\begin{array}{c}\text { Non-Guassian } \\
\text { (moment-based } \\
\text { translation model) }\end{array}$ & $\begin{array}{c}\text { Non-Guassian } \\
\text { (CDF-based } \\
\text { translation model) }\end{array}$ \\
\hline Tap 206 & 16.1 & 0.52 & 0.32 & 0.35 & $17.0(6 \%)$ & $17.4(8 \%)$ & $17.3(8 \%)$ \\
Tap 44 & 38.3 & 0.55 & 0.36 & 0.37 & $37.2(3 \%)$ & - & $40.3(5 \%)$ \\
Tap 28 & 45.8 & 0.47 & 0.27 & 0.33 & $74.8(63 \%)$ & $48.7(6 \%)$ & $48.5(6 \%)$ \\
Tap 112 & 3.2 & 0.61 & 0.41 & 0.43 & $2.6(20 \%)$ & $3.5(7 \%)$ & $3.5(7 \%)$ \\
Tap 36 & 13.3 & 0.67 & 0.48 & 0.47 & $8.1(39 \%)$ & $17.4(31 \%)$ & $14.6(10 \%)$ \\
Tap 31 & 94.6 & 0.50 & 0.29 & 0.36 & $108(14 \%)$ & $78.7(17 \%)$ & $99.8(5 \%)$ \\
\hline
\end{tabular}




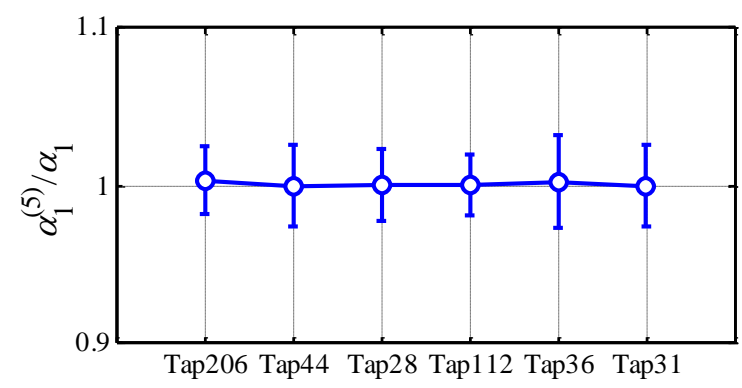

(a) $\alpha_{1}$

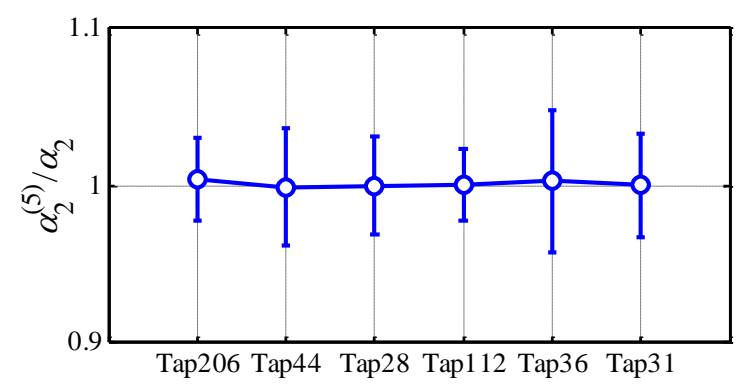

(b) $\alpha_{2}$

Fig. 15 Bandwidth parameters and its $95 \%$ confidence intervals from 5 short-term samples

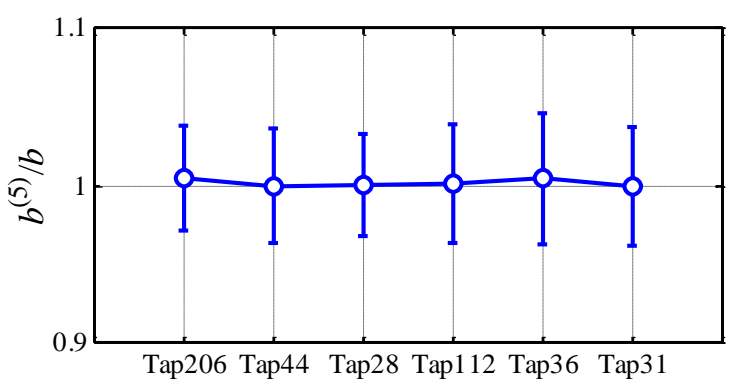

Fig. $16 b$ value and its $95 \%$ confidence intervals from 5 short-term samples

The deficiency of moment-based translation model can be overcome by directly curve-fitting the numerical translation function derived from CDF mapping, which is referred to as CDF-based translation model. In the case of softening non-Gaussian processes, a constrained least-square optimization is conducted, where the constraints are used to ensure the monotonic increasing of translation function (Ding and Chen 2014)

$$
\sum_{i=1}^{n}\left|g\left(x_{i}\right)-\kappa\left[x_{i}+h_{3}\left(x_{i}^{2}-1\right)+h_{4}\left(x_{i}^{3}-3 x_{i}\right)\right]\right|^{2}
$$

Subject to: $h_{4}>0$ and $h_{3}^{2}-3 h_{4}\left(1-3 h_{4}\right)<0$

In the case of hardening non-Gaussian processes, they are

$$
\sum_{i=1}^{n}\left|g^{-1}\left(z_{i}\right)-\left[b_{2} z_{i}+b_{3}\left(z_{i}^{2}-\gamma_{3} z_{i}-1\right)+b_{4}\left(z_{i}^{3}-\gamma_{4} z_{i}-\gamma_{3}\right)\right]\right|^{2}
$$

Subject to: $b_{4}>0$ and $b_{3}^{2}-3 b_{4}\left(b_{2}-b_{3} \gamma_{3}-b_{4} \gamma_{4}\right)<0$ 


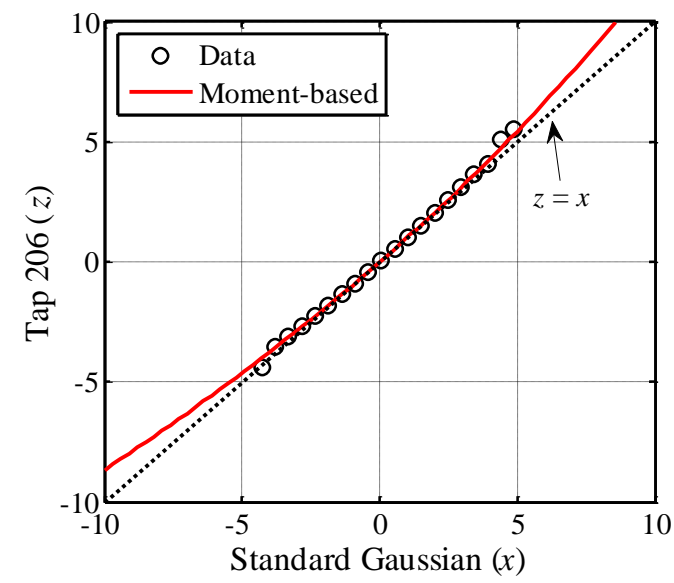

(a) Tap $206\left(\gamma_{3}=0.10 ; \gamma_{4}=3.05\right)$

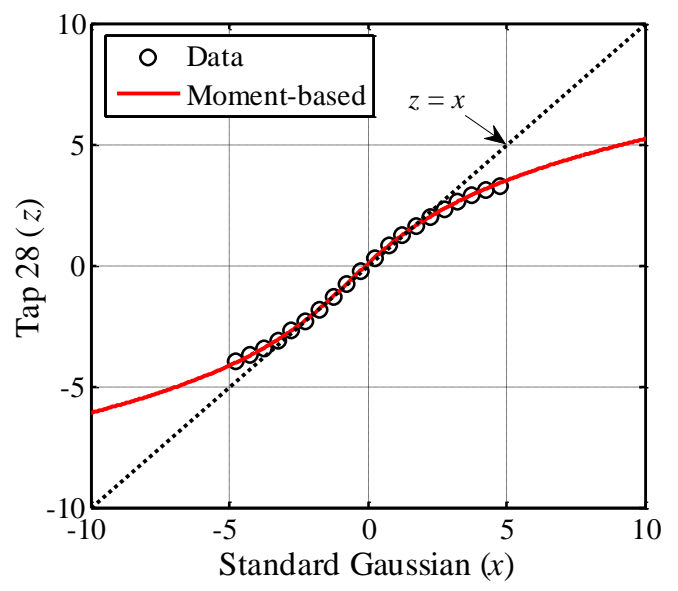

(c) Tap $28\left(\gamma_{3}=-0.20 ; \gamma_{4}=2.65\right)$

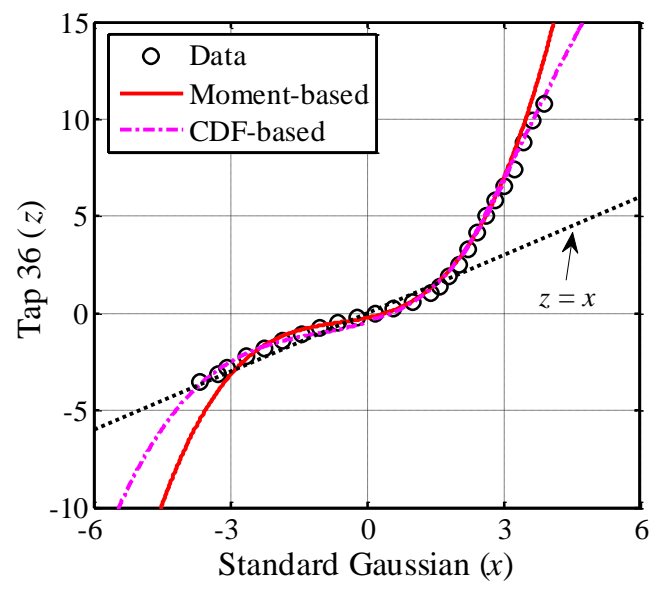

(e) $\operatorname{Tap} 36\left(\gamma_{3}=2.22 ; \gamma_{4}=14.7\right)$

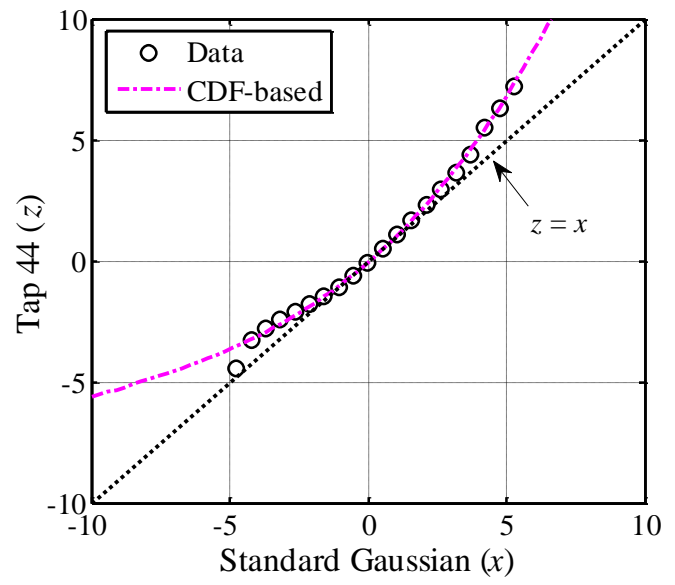

(b) Tap $44\left(\gamma_{3}=0.42 ; \gamma_{4}=3.04\right)$

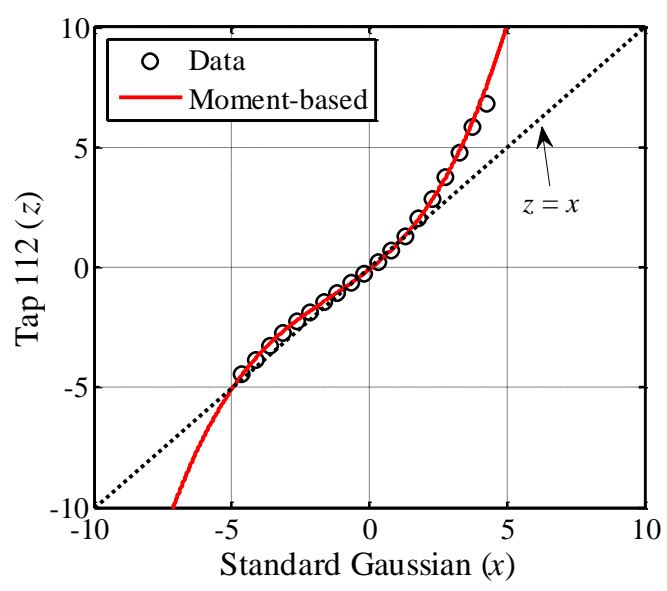

(d) Tap $112\left(\gamma_{3}=0.70 ; \gamma_{4}=4.35\right)$

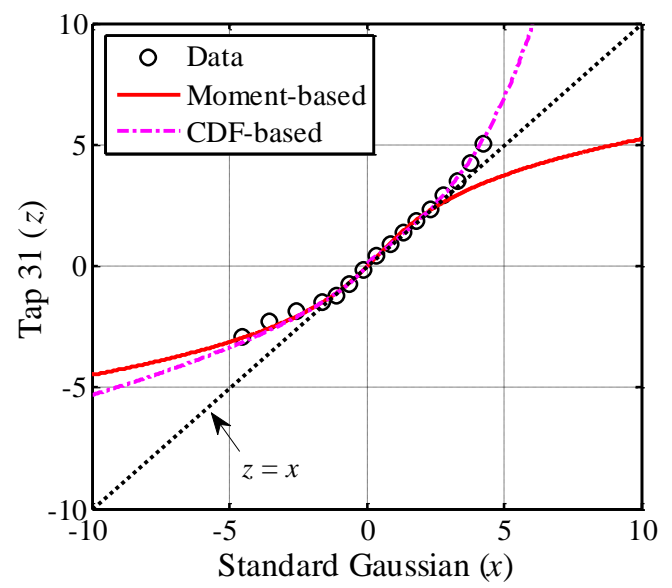

(f) Tap 31 (Bimodal distribution)

Fig. 17 Comparison of translation functions 
It is noted that the positive and negative tails of the numerical translation function can show different properties. For instance, the positive part of translation function for the process of Tap 31 shows softening non-Gaussian properties, while the negative part shows hardening non-Gaussian properties. In such a case, different translation models are applied in modeling these two parts separately. The CDF-based translation models are also shown in Fig. 17, which generally have a better performance. The CDF-based translation function model can also resolve the applicability limit of moment-based model as shown in Fig. 17(b).

Fig. 18 shows the comparison of fatigue loading spectra estimated from TB spectral method and rainflow counting method. The fatigue loading spectrum is the distribution of amplitudes in terms of probability of exceedance:

$$
F(s)=\int_{s}^{+\infty} p_{S}(u) d u
$$

The fatigue loading spectrum for rainflow counting method is directly constructed from the amplitudes extracted from all short-term samples, which is used as a baseline for comparison. For TB spectral method with Gaussian assumption, it is given as the following closed-form:

$$
F_{T B}(s)=v_{p}\left[b \alpha_{2} e^{-\frac{s^{2}}{2 \sigma_{X}^{2}}}+(1-b) e^{-\frac{s^{2}}{2 \sigma_{X}^{2} \alpha_{2}^{2}}}\right]
$$

The fatigue loading spectrum of a non-Gaussian process is evaluated through a numerical integration. The estimated fatigue damage is summarized in Table 3. The proposed expression $b_{\text {new }}$ is adopted in all applications. It is observed that the TB spectral method with adequately modeled translation function can result in satisfactory estimation of non-Gaussian fatigue as compared to that from rainflow counting method. For near Gaussian process with kurtosis close to 3 but noticeable skewness, i.e., Taps 206 and 44, the TB spectral method with Gaussian assumption is sufficient. However, the Gaussian assumption overestimates the fatigue damage of the hardening non-Gaussian process, i.e., Tap 28, and underestimates that of the softening non-Gaussian process, i.e., Taps 112 and 36. The performance of the non-Gaussian TB spectral method greatly depends on the goodness of the translation function models. 


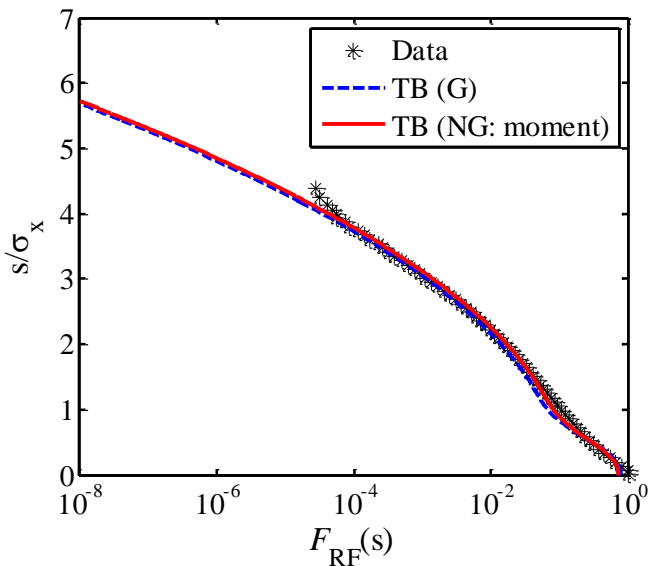

(a) Tap $206\left(\gamma_{3}=0.10 ; \gamma_{4}=3.05\right)$

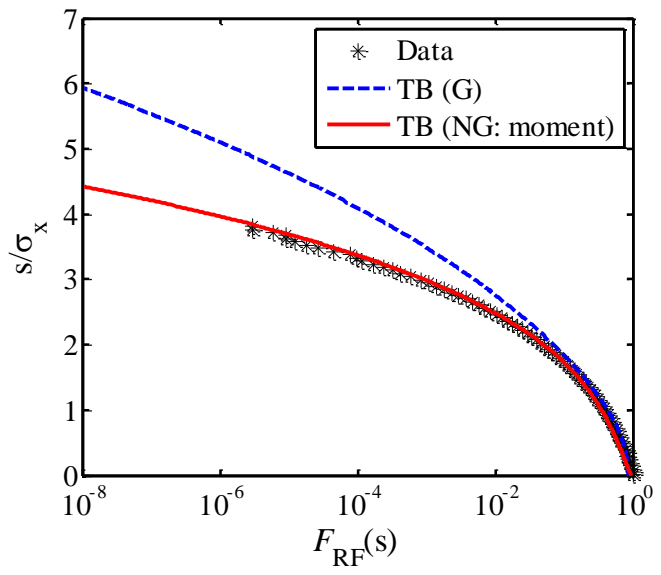

(c) Tap $28\left(\gamma_{3}=-0.20 ; \gamma_{4}=2.65\right)$

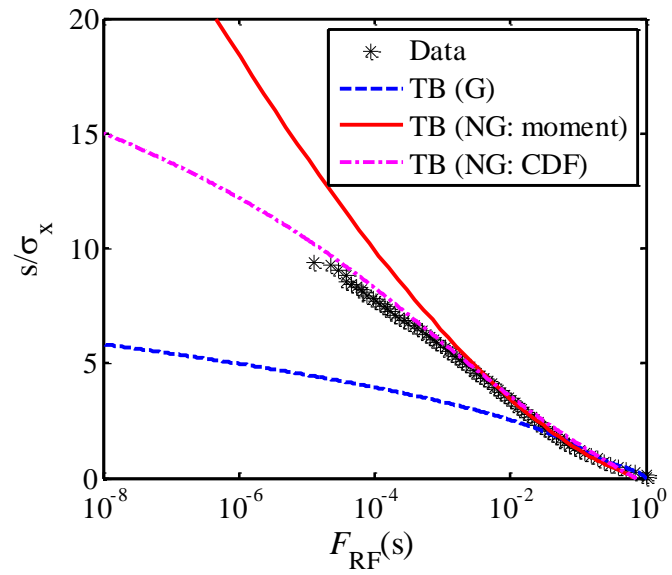

(e) $\operatorname{Tap} 36\left(\gamma_{3}=2.22 ; \gamma_{4}=14.7\right)$

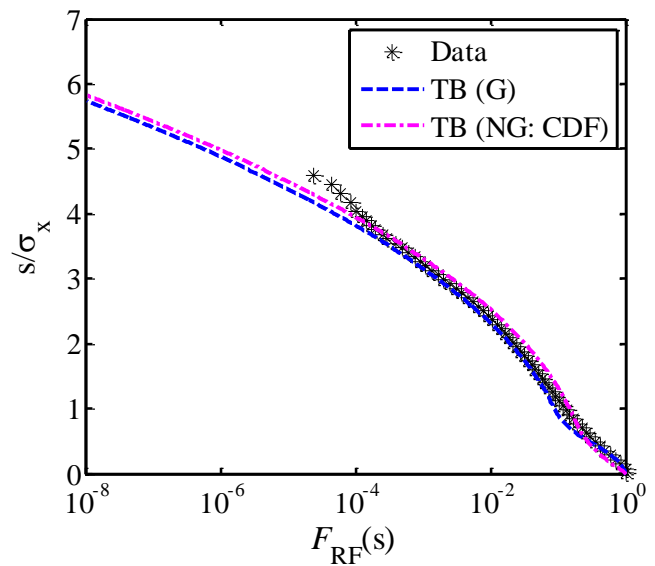

(b) Tap $44\left(\gamma_{3}=0.42 ; \gamma_{4}=3.04\right)$

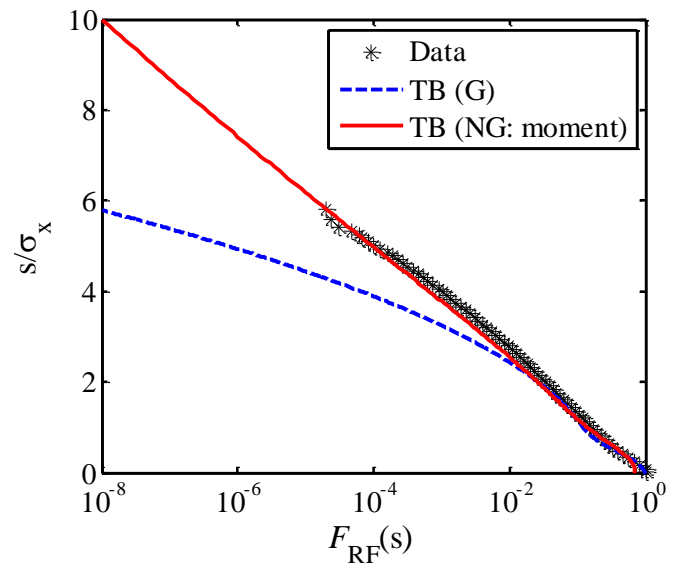

(d) Tap $112\left(\gamma_{3}=0.70 ; \gamma_{4}=4.35\right)$

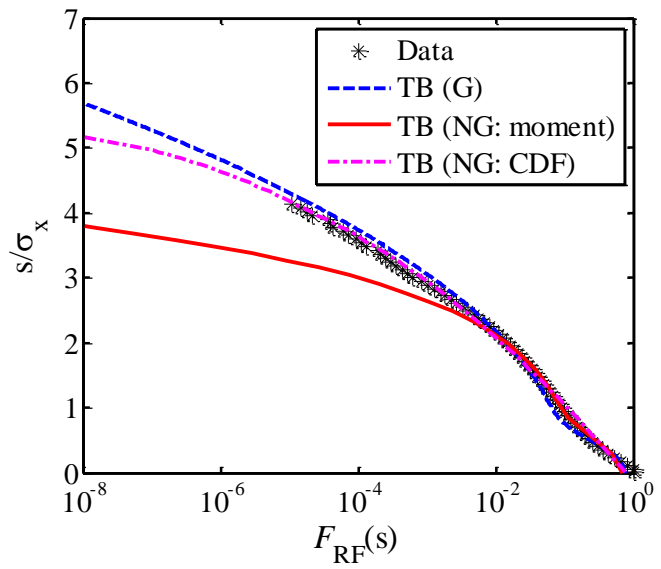

(f) Tap 31 (Bimodal distribution)

Fig. 18 Fatigue loading spectra for different processes 


\section{Concluding remarks}

The TB spectral method was re-evaluated for fatigue analysis of broad-band Gaussian and nonGaussian wind load effects. This method approximates the rainflow counting damage as a linear combination of its upper and lower bounds estimated from level-crossing and range-mean counting methods. The combination factor depends on the bandwidth parameters of the process power spectrum in terms of its four moments. Applications of this method for alongwind, crosswind and their coupled responses of tall buildings, and wind pressures on claddings illustrated its effectiveness through comparison with rainflow counting damage estimated from time history samples. The newly proposed combination factor resulted in a better prediction of fatigue damage of broad-band Gaussian wind load effects as compared to the original TB spectral method.

The TB spectral method facilitates fatigue analysis of broad-band non-Gaussian processes which are modeled as translation processes from the underlying Gaussian processes. The effectiveness and accuracy of this spectral method for non-Gaussian fatigue damage evaluation was demonstrated through a set of synthetic and experimental pressure data. The results illustrated that the influences of non-Gaussian distribution and broad-band feature on fatigue damage can be treated separately. The combination factor used in Gaussian fatigue can also be used for non-Gaussian fatigue damage evaluation. The translation function model which relates the non-Gaussian process with underlying Gaussian process is essential for non-Gaussian fatigue damage evaluation. It was also demonstrated that the moment-based translation function model is only applicable to mildly non-Gaussian processes and the translation model determined by a direct curve-fitting is more effective for processes with a wide range of non-Gaussian characteristics, including processes beyond the application region of moment-based model, and processes whose first several statistical moments are not sufficient in representing the probability distribution.

\section{Acknowledgments}

The support for this work provided in part by NSF Grant No. CMMI-1029922 is greatly acknowledged. 


\section{Appendix A: The PSDs of alongwind and crosswind base bending moment coefficients $C_{M}(t)$ of tall}

\section{buildings}

The PSD of alongwind base bending moment coefficient $C_{M}(t)$ is determined based on Architectural Institute of Japan (AIJ) recommendations (AIJ 2004), which is expressed as the product of aerodynamic admittance function and wind fluctuation spectrum:

$$
\begin{gathered}
f S_{C_{M}}(f) / \sigma_{C_{M}}^{2}=\frac{0.90 F_{D}}{\left[1+6\left(\frac{f H}{U_{H}}\right)^{2}\right]^{0.5}\left(1+3 f B / U_{H}\right)} \times \frac{4\left(f L_{H} / U_{H}\right)}{\left[1+71\left(\frac{f L_{H}}{U_{H}}\right)^{2}\right]^{5 / 6}} \\
F_{D}=1+\frac{0.87}{1+20\left(\frac{f B}{U_{H}}\right)}
\end{gathered}
$$

where $C_{M}(t)=M(t) /\left(0.5 \rho U_{H}^{2} B H^{2}\right) ; \rho=1.29 \mathrm{~kg} / \mathrm{m}^{3}$ is air density; $U_{H}$ is the mean wind speed at building top; $f$ is frequency in $\mathrm{Hz} ; L_{H}=100 \sqrt{H / 30}$ is the turbulence length scale; and $\sigma_{C_{M}}$ is rootmean-square (RMS) of $C_{M}(t)$ in alongwind direction and is taken as 0.11 for this building; $F_{D}$ is a coefficient calculated with an assumption that the exponent of power law in wind speed profile is 0.35 for the urban environment (Zhou et al. 2003).

The PSD function of crosswind base bending moment coefficient suggested by AIJ recommendations is:

$$
\begin{gathered}
f S_{C_{M}}(f) / \sigma_{C_{M}}^{2}=\sum_{j=1}^{N} \frac{4 \kappa_{j}\left(1+0.6 \beta_{j}\right) \beta_{j} \times\left(f / f_{s j}\right)^{2}}{\pi \times\left\{\left[1-\left(f / f_{s j}\right)^{2}\right]^{2}+4 \beta_{j}^{2}\left(f / f_{s j}\right)^{2}\right\}} \\
N=\left\{\begin{array}{l}
1, D / B<3 \\
2, D / B>3
\end{array} \text { and } \kappa_{1}=0.85, \kappa_{2}=0.02\right.
\end{gathered}
$$

where $\beta_{j}$ is related to the bandwidth; $f_{s j}$ represents the peak frequencies of the spectrum, i.e., vortexshedding frequencies; $D$ is the building depth; $\sigma_{C_{M}}$ is the RMS base bending moment coefficient in crosswind direction. These parameters are determined by following empirical formulae as functions of the building side ratio $(D / B)$ :

$$
\sigma_{C_{M}}=0.0082(D / B)^{3}-0.071(D / B)^{2}+0.22(D / B)
$$




$$
\begin{gathered}
f_{S 1}=\frac{0.12}{\left[1+0.38(D / B)^{2}\right]^{0.89}} \frac{U_{H}}{B} \\
f_{S 2}=\frac{0.56}{(D / B)^{0.85}} \frac{U_{H}}{B} \\
\beta_{1}=\frac{(D / B)^{4}+2.3(D / B)^{2}}{2.4(D / B)^{4}-9.2(D / B)^{3}+18(D / B)^{2}+9.5(D / B)-0.15}+\frac{0.12}{(D / B)} \\
\beta_{2}=0.28(D / B)^{-0.34}
\end{gathered}
$$

\section{Appendix B: Closed-form formulations for calculating the spectral moments of alongwind displacement of tall buildings}

The following closed-form formulation can be used for calculating the spectral moments of

alongwind displacement of a tall building (e.g., Vanmarcke 1972; Der Kiureghian 1980; Chen and Kareem 2005c):

$$
\begin{gathered}
\lambda_{0}=\lambda_{0 b}+\lambda_{0 r} \\
\lambda_{0 b}=\frac{\lambda_{0 Q}}{M_{1}^{2}\left(2 \pi f_{1}\right)^{4}} ; \quad \lambda_{0 r}=\frac{1}{M_{1}^{2}\left(2 \pi f_{1}\right)^{4}} \frac{\pi f_{1} S_{Q}\left(f_{1}\right)}{4 \zeta} \\
\lambda_{1}=\lambda_{1 b}+\lambda_{1 r} \\
\lambda_{1 b}=\frac{\lambda_{1 Q}}{\lambda_{0 Q}} \lambda_{0 b} ; \quad \lambda_{1 r}=\frac{\left(2 \pi f_{1}\right) \lambda_{0 r}}{\sqrt{1-\zeta^{2}}}\left[1-\frac{2}{\pi} \tan ^{-1}\left(\frac{\zeta}{\sqrt{1-\zeta^{2}}}\right)\right] \\
\lambda_{0 Q}=\sigma_{Q}^{2}=\int_{0}^{\infty} S_{Q}(f) d f ; \lambda_{1 Q}=\int_{0}^{\infty}(2 \pi f) S_{Q}(f) d f \\
\lambda_{2}=\left(2 \pi f_{1}\right)^{2} \lambda_{0 r} \\
\lambda_{4}=\left(2 \pi f_{1}\right)^{4} \lambda_{0 r}
\end{gathered}
$$

where $S_{Q}(f)$ is the PSD of generalized force $Q(t) ; S_{Q}\left(f_{1}\right)$ is the corresponding value at fundamental frequency of the structure $f_{1} ; \lambda_{i b}$ and $\lambda_{i r}(i=0,1)$ denote the $i$-th spectral moment from background and 
resonant responses, respectively; $\sqrt{\lambda_{0 b}}$ and $\sqrt{\lambda_{0 r}}$ are equal to the RMS background and resonant responses. It is noted that the contributions of background response to higher spectra moments are small and thus neglected.

Accordingly, the mean crossing rate and bandwidth parameters are estimated as

$$
\begin{gathered}
v_{0}=\frac{f_{1}}{\sqrt{1+\chi_{0}}} \\
\alpha_{1}=\frac{1}{\sqrt{1-\zeta^{2}}}\left[1-\frac{2}{\pi} \tan ^{-1}\left(\frac{\zeta}{\sqrt{1-\zeta^{2}}}\right)\right] \frac{\left(1+\chi_{1}\right)}{\sqrt{1+\chi_{0}}} \\
\alpha_{2}=\frac{1}{\sqrt{1+\chi_{0}}}
\end{gathered}
$$

where $\chi_{0}=\lambda_{0 b} / \lambda_{0 r} ; \chi_{1}=\lambda_{1 b} / \lambda_{1 r}$. It is noted when the background responses is negligible small and modal damping is very low, we have $v_{0} \approx f_{1}, \alpha_{1} \approx 1-2 \zeta / \pi$ and $\alpha_{2} \approx 1$. The accuracy of those closedform formulations has been verified in this study. 


\section{References}

[1] Architectural Institue of Japan (2004). AIJ recommendations for load on buildings, AIJ, Tokyo.

[2] ASTM 1049-85 (2011). Standard practices for cycle counting in fatigue analysis. ASTM International.

[3] Bouyssy, V., Naboishikov, S.M., and Rackwitz, R. (1993). "Comparison of analytical counting methods for Gaussian processes.” Structural Safety, 12, 35-57.

[4] Benasciutti, D., and Tovo, R. (2005). "Cycle distribution and fatigue damage assessment in broadband non-Gaussian random processes.” Probabilistic Engineering Mechanics, 20, 115-127.

[5] Benasciutti, D., and Tovo, R. (2006). "Comparison of spectral methods for fatigue analysis of broadband Gaussian random process.” Probabilistic Engineering Mechanics, 21, 287-299.

[6] Chen, X. (2014a). "Extreme value distribution and peak factor of crosswind response of flexible structures with nonlinear aeroelastic effect." Journal of Structural Engineering, ASCE, 04014091.

[7] Chen, X. (2014b). "Analysis of crosswind fatigue of wind-excited structures with nonlinear aerodynamic damping." Engineering Structures, 74, 145-156.

[8] Chen, X., and Kareem, A. (2005a). "Dynamic wind effects on buildings with 3D coupled modes: application of high frequency force balance measurements." Journal of Engineering Mechanics, ASCE, 131(11), 1115-1125.

[9] Chen, X., Kareem, A. (2005b). "Proper orthogonal decomposition-based modeling, analysis, and simulation of dynamic wind load effects on structures." Journal of Engineering Mechanics, ASCE, 131(4), 325-339.

[10] Chen, X., and Kareem, A. (2005c). "Coupled dynamic analysis and equivalent static wind loads on buildings with three-dimensional modes." Journal of Structural Engineering, ASCE, 131(7), 10711082.

[11] Davenport, A.G. (1964). "Note on the distribution of the largest value of a random function with application to gust loading." Proceedings of Institution of Civil Engineers, pp187-196.

[12] Der Kiureghian, A. (1980). "Structural response to stationary excitation." Journal of Engineering Mechanics Division, 106(6), 1195-1213.

[13] Ding, J., and Chen, X. (2013). "Assessing small failure probability by importance splitting method and its application to wind turbine extreme response prediction". Engineering Structures, 54, 180-191.

[14] Ding, J., and Chen, X. (2014). "Assessment of methods for extreme value analysis of non-Gaussian wind effects with short-term time history samples." Engineering Structures, 80, 75-88.

[15] Ding, J., and Chen, X. (2015). "Moment-based translation model for hardening non-Gaussian response processes." Journal of Engineering Mechanics, ASCE (Under review).

[16] Dirlik, T. (1985). Application of computers in fatigue analysis. Ph.D. thesis. UK: University of Warwich.

[17] Dowling, N.E. (1972). "Fatigue failure predictions for complicated stress-strain histories." Journal of Materials, 7(1), 71-87.

[18] Gao Z., and Moan, T. (2007). "Fatigue damage induced by non-Gaussian bimodal wave loading in mooring lines." Applied Ocean Research, 29, 45-54.

[19] Gao, Z., and Moan, T. (2008). "Frequency-domain fatigue analysis of wide-band stationary Gaussian processes using a trimodal spectral formulation.” International Journal of Fatigue, 30, 1944-1955. 
[20] Gong, K., Ding, J., and Chen, X. (2014). "Estimation of long-term extreme response of operational and parked wind turbines: Validation and some new insights." Engineering Structures (in press).

[21] Grigoriu, M. (1984). "Crossings of non-Gaussian translation processes." Journal of Engineering Mechanics, ASCE, 110(4), 610-620.

[22] Grigoriu, M. (1995). Applied non-Gaussian processes: examples, theory, simulation, linear random vibration, and matlab solution. Prentice Hall, Englewood Cliffs, NJ.

[23] Holmes, J.D. (1981). "Non-Gaussian characteristics of wind pressure fluctuations." Journal of Wind Engineering and Industrial Aerodynamics, 7(1), 103-108.

[24] Holmes, J.D. (2002). "Fatigue life under along-wind loading: closed-form solutions." Engineering Structures, 24, 109-114.

[25] Jiao, G., and Moan, T. (1990). "Probabilistic analysis of fatigue due to Gaussian load processes." Probabilistic Engineering Mechanics. 5(2), 76-83

[26] Kumar, K.S., and Stathopoulos, T. (1998). "Fatigue analysis of roof cladding under simulated wind loading." Journal of Wind Engineering and Industrial Aerodynamics, 77-78, 171-183.

[27] Lin, N., Letchford, C., Tamura, Y., Liang, B., and Nakamura, O. (2005). "Characteristics of wind forces acting on tall buildings." Journal of Wind Engineering and Industrial Aerodynamics, 93, 217242.

[28] Lutes, L.D., Corazao, M., Hu, S., and Zimmerman, J. (1984). "Stochastic fatigue damage accumulation." Journal of Structural Engineering, ASCE. 110(11), 2585-2601.

[29] Lutes, L.D., Larsen, C.E. (1990). "Improved spectral methods for variable amplitude fatigue." Journal of Structural Engineering, ASCE. 116(4), 1149-1164.

[30] Lutes, L.D., and Sarkani, S. (2004). Random vibrations: Analysis of structural and mechanical systems. Elsevier Butterworth-Heinemann, MA.

[31] Lynn, B.A., and Stathopoulos, T. (1985). "Wind-induced fatigue on low metal buildings." Journal of Structural Engineering, ASCE. 111(4), 826-839.

[32] Madsen, H.O., Krenk, S., and Lind, N.C. (1986). Methods of structural safety, Prentice-Hall, Englewood Cliffs, NJ.

[33] Repetto, M.P., and Solari, G. (2004). "Directional wind-induced fatigue of slender vertical structures." Journal of Structural Engineering, ASCE. 130(7), 1032-1040.

[34] Repetto, M.P., and Solari, G. (2006). "Bimodal alongwind fatigue of structures." Journal of Structural Engineering, ASCE. 132(6), 899-908.

[35] Rychlik, I. (1993). "Note on cycle counts in irregular loads." Fatigue \& Fracture of Engineering Materials and Structures, 16(4), 377-390.

[36] Shinozuka, M., and Jan, C.M. (1972). "Digital simulation of random processes and its applications." Journal of Sound and Vibration, 25(10), 111-128.

[37] Simiu, E., and Scanlan, R.H. (1996). Wind effects on structures: Fundamentals and applications to design, $3^{\text {rd }}$ Edition, John Wiley \& Sons, New York.

[38] Tovo, R. (2002). "Cycle distribution and fatigue damage under broad-band random loading." International Journal of Fatigue, 24, 1137-1147.

[39] Vanmarcke, E. H. (1972). "Properties of spectral moments with applications to random vibration." Journal of Engineering Mechanics Division, 98(2), 425-446. 
[40] Winterstein, S.R. (1988). "Nonlinear vibration models for extremes and fatigue." Journal of Engineering Mechanics, ASCE 114(10), 1772-1790.

[41] Winterstein, S.R., and Kashef, T. (2000). "Moment-based load and response models with wind engineering applications." Journal of Solar Energy Engineering, 122(3), 122-128.

[42] Winterstein, S.R., and MacKenzie, C.A. (2012). "Extremes of nonlinear vibration: models based on moments, L-moments, and maximum Entropy." Journal of Offshore Mechanics and Arctic Engineering, 135(2), 021602.

[43] Wirsching, P.H., and Light, C.L. (1980). "Fatigue under wide band rondom stresses." Journal of structural Division, ASCE. 106(7), 1593-1607.

[44] Xu, Y.L. (1995). "Determination of wind-induced fatigue loading on roof cladding." Journal of Engineering Mechanics, ASCE. 121(9), 956-963.

[45] Yeatts, B.B., and Metha, K.C. (1993). "Field experiments for building aerodynamics." Journal of Wind Engineering and Industrial Aerodynamics, 50, 213-224.

[46] Zhao, W., and Baker, M.J. (1990). "A new stress range distribution model for fatigue analysis under wave loading." Environmental Forces of Offshore Structures and Their Prediction. Kluwer Academic Publishers, 271-291.

[47] Zhou, Y., Kijewski, T., and Kareem, A. (2003). "Aerodynamic loads on tall buildings: interactive database." Journal of Structural Engineering, ASCE.129(3), 394-404. 